\title{
A Machine Learning-Driven Evolutionary Approach for Testing Web Application Firewalls
}

\author{
Dennis Appelt, Cu D. Nguyen, Annibale Panichella, Lionel C. Briand Fellow, IEEE
}

\begin{abstract}
Web application firewalls (WAF) are an essential protection mechanism for online software systems. Because of the relentless flow of new kinds of attacks as well as their increased sophistication, WAFs have to be updated and tested regularly to prevent attackers from easily circumventing them. In this paper, we focus on testing WAFs for SQL injection attacks, but the general principles and strategy we propose can be adapted to other contexts. We present ML-Driven, an approach based on machine learning and an evolutionary algorithm to automatically detect holes in WAFs that let SQL injection attacks bypass them.

Initially, ML-Driven automatically generates a diverse set of attacks and submit them to the system being protected by the target WAF. Then, ML-Driven selects attacks that exhibit patterns (substrings) associated with bypassing the WAF and evolve them to generate new successful bypassing attacks. Machine learning is used to incrementally learn attack patterns from previously generated attacks according to their testing results, i.e., if they are blocked or bypass the WAF. We implemented ML-Driven in a tool and evaluated it on ModSecurity, a widely used open-source WAF, and a proprietary WAF protecting a financial institution. Our empirical results indicate that ML-Driven is effective and efficient at generating SQL injection attacks bypassing WAFs and identifying attack patterns.
\end{abstract}

Index Terms-Software Security Testing, SQL Injection, Web Application Firewall.

\section{INTRODUCTION}

W EB application firewalls (WAF) protect enterprise web systems from malicious attacks. As a facade to the web application they protect, WAFs inspect incoming HTTP messages and decide whether blocking or forwarding them to the target web application. The decision is often performed based on a set of rules, which are designed to detect attack patterns. Since cyber-attacks are increasingly sophisticated, WAF rules tend to become complex and difficult to manually maintain and test. Therefore, automated testing techniques for WAFs are crucial to prevent malicious requests from reaching web applications and services.

In this work, we focus our testing efforts on a common category of attacks, namely SQL injections (SQLi). SQLi has received a lot of attention from academia as well as practitioners [7], [10], [14], [24], [25], [26], [27], [32], [34], [45]. Yet

- Dennis Appelt, Cu D. Nguyen, Annibale Panichella, and Lionel C. Briand are with the SnT Centre, University of Luxembourg, L-2721 Luxembourg, Luxembourg.

Manuscript received March xx, 2016; revised yyyyyy xx, 2016. the Open Web Application Security Project (OWASP) finds that the prevalence of SQLi vulnerabilities is common and the impact of a successful exploitation is severe [50]. While we assess our approach based on the example of SQLi, we believe that many of the principles of our methodology can be adapted to other forms of attacks.

Various techniques have been proposed in the literature to detect SQLi attacks based on a variety of approaches, including white-box testing [21], static analysis [20], modelbased testing [30], and black-box testing [18]. However, such techniques present some limitations which may adversely impact their practical applicability as well as their vulnerability detection capability. For example, white-box testing techniques and static analysis tools require access to source code [33], which might not be possible when dealing with third-party components or industrial appliances, and are linked to specific programming languages [19]. Modelbased testing techniques require models expressing the security policies or the implementation of WAFs and the web application under test [30], which are often not available or very difficult to manually construct. Black-box testing strategies do not require models or the source code but they are less effective in detecting SQLi vulnerabilities. Indeed, comprehensive reviews on black-box techniques [13], [18] have revealed that many types of security vulnerabilities (including SQLi attacks) remain largely undetected and, thus, warrant further research.

In our preliminary work [9], we introduced a novel black-box technique, namely ML-Driven, that combines the classical $(\mu+\lambda)$ evolutionary algorithm (EAs) with machine learning algorithms for generating tests (i.e., attacks) bypassing a WAF's validation routines. ML-Driven uses machine learning to incrementally learn attack patterns and build a classifier, i.e., that predicts combinations of attack substrings ("slices") associated with bypassing the WAF. The resulting classifier is used within the main loop of $(\mu+\lambda)$-EAs to rank tests depending on which substrings compose them and the corresponding bypassing probabilities. In each iteration, tests with the highest rank are selected and mutated to generate $\lambda$ new tests (offsprings), which are then executed against the WAF. The corresponding execution results are used to re-train the classifier to incrementally improve its accuracy. Through subsequent generations, tests are evolved to increase the number of attacks able to bypass the target WAF.

We defined two variants of ML-Driven, namely 
ML-Driven D and ML-Driven B, that differ in the number of tests being selected for generating new tests (offsprings). The former variant selects fewer tests to evolve but generates more offsprings per selected test, thus increasing $e x$ ploitation (deep search). The latter variant selects more tests to mutate, which results in a lower number of offsprings per selected test, hence increasing exploration (broad search). Our preliminary study with ModSecurity ${ }^{1}$ a popular open source WAF, has shown that both ML-Driven D and ML-Driven $B$ are effective in generating a large number of distinct SQLi attacks passing thought the WAF. However, $M L-D r i v e n ~ D$ performs better in the earlier stages of the search while ML-Driven B outperforms it in the last part of the search, for reasons we will explain.

In the race against cyber-attacks, time is vital. Being able to learn and anticipate attacks that successfully bypass WAFs in a timely manner is critical. With more successful, distinct attacks being detected, the WAF administrator is in a better position to identify missed attack patterns and to devise patches that block all further attacks sharing the same patterns. Therefore, our goal is to devise a technique that can efficiently generate as many successful distinct attacks as possible. To this aim, in this paper we extended our prior work and propose an adaptive variant of ML-Driven, namely ML-Driven E (Enhanced), that combines the strengths of ML-Driven D (deep search) and ML-Driven B (broad search) in an adaptive manner. In ML-Driven E, the number of offsprings is computed dynamically depending on the bypassing probability assigned to each selected test by the constructed classifier. Conversely, $\mathrm{ML}-\mathrm{Driven} \mathrm{B}$ and D generate a fixed number of offsprings per attack/test. Therefore, ML-Driven $\mathrm{E}$ is a more flexible approach that better balances exploration over run time.

Moreover, we conducted a much larger empirical study with two popular WAFs that protect three open-source and 44 proprietary web services. The results show that the enhanced version of our technique (ML-Driven E) significantly outperforms: (i) its predecessors ML-Driven B and ML-Driven D; (ii) a random test strategy, which serves as a baseline; (iii) two state-of-the-art vulnerability detection tools, namely SqlMap and WAF Testing Framework. We also performed a qualitative analysis of the attacks generated by our approach and we found out that they enable the identification of attack patterns that are strongly associated with bypassing the WAFs, thus providing better support for improving the WAFs' rule set.

To summarize, the key contributions of this paper include:

- Enhancing ML-Driven with an adaptive test selection and generation heuristic, which more effectively explores attack patterns with higher likelihood of bypassing the WAF. This enhanced ML-Driven variant is intended to replace its predecessors, leaving the practitioners with a single, yet the best, option.

- Assessing the influence of the selected machine learning algorithm on the test results by comparing two alternative classification models, namely RandomTree and RandomForest, which are both adapted

1. https://www.modsecurity.org to large numbers of features and datasets, but with complementary advantages and drawbacks.

- Extending the previous evaluation and conducting a large-scale experiment on a proprietary WAF protecting a financial institution.

- Comparing ML-Driven with SqlMap and WAF Testing Framework, which are state-of-the-art vulnerability detection tools.

- A qualitative analysis showing that the additional distinct attacks found by ML-Driven E help security analysts design better patches compared to the other ML-Driven variants, RAN, and state-of-theart vulnerability detection tools.

The remainder of the paper is structured as follows. Section 2 provides background information on WAFs as well as SQLi attacks and discusses related work. Section 3 details our approach followed by Section 4 where we describe the design and procedure of our empirical evaluation. Section 5 describes the evaluation results and their implications regarding our research questions while Section 6 explains and illustrates how to use the generated attacks for repairing vulnerable WAFs. Further reflections on the results are provided in Section 7 while Section 8 concludes this paper.

\section{Backround AND ReLated Work}

In this section, we provide background notions about SQLi vulnerabilities and describe existing white-box and blackbox approaches aimed at uncovering them.

\subsection{SQL Injection Vulnerabilities}

In systems that use databases, such as web-based systems, the SQL statements accessing back-end databases are usually treated as strings. These strings are formed by concatenating different string fragments based on user choices or the application's control flow. Once a SQL statement is formed, it is submitted to the database server to be executed. For example, a SQL statement can be formed as follows (a simplified example from one of our web services in the case study):

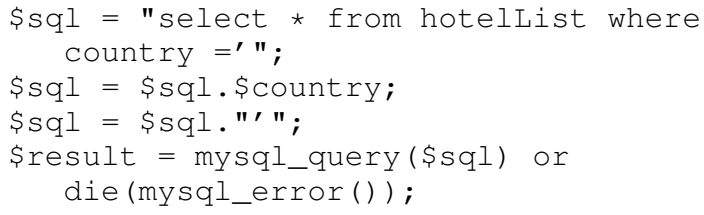

The variable \$country is an input provided by the user, which is concatenated with the rest of the SQL statement and then stored in the string variable $\$ s q l$. The string is then passed to the function mysql_query that sends the SQL statement to the database server to be executed.

SQLi is an attack technique in which attackers inject malicious SQL code fragments into input parameters that lack proper validation or sanitization. An attacker might construct input values in a way that changes the behavior of the resulting SQL statement and performs arbitrary actions on the database (e.g. exposure of sensitive data, insertion or alteration of data without authorization, loss of data, or even taking control of the database server). 
In the previous example, if the input \$country received the attack payload ' or $1=1--$, the resulting SQL statement is:

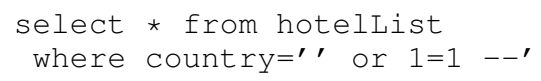

The clause or $1=1$ is a tautology, i.e., the condition will always be true, and is thus able to bypassing the original condition in the where clause, making the SQL query return all rows in the table.

Web Application Firewalls. Web applications with high security requirements are commonly protected by WAFs. In the overall system architecture, a WAF is placed in front of the web application that has to be protected. Every request that is sent to the web application is examined by the WAF before it reaches the web application. The WAF hands over the request to the web application only if the request complies with the firewall's rule set.

A common approach to define the firewall's rule set is using a black-list. A black-list contains string patterns, typically defined as regular expressions. Requests recognized by these patterns are likely to be malicious attacks (e.g., SQLi) and, therefore, are blocked. For example, the following regular expression describes the syntax for SQL comments, e.g., $/ * \star /$ or \#, which are frequently used in SQLi attacks:

$$
\begin{aligned}
八 \star & ! ?|\backslash * /|\left[{ }^{\prime} ;\right]--\mid--[\backslash S \backslash r \backslash n \backslash v \backslash f] \\
\mid & (?:--[\wedge-] * ?-) \\
\mid & ([\wedge \backslash-\&]) \# . * ?[\backslash S \backslash r \backslash n \backslash v \backslash f] \mid ; ? \backslash \backslash x 00
\end{aligned}
$$

There are several reasons why a WAF may provide insufficient protection, including implementation bugs or misconfiguration. One way to ensure the resilience of a WAF against attacks is to rely on an automated testing procedure that thoroughly and efficiently detects vulnerabilities. This paper addresses this challenge for SQL injections, one of the main types of attacks in practice.

\subsection{Related Work}

Previous research on ensuring the resilience of IT systems against malicious requests has focused on the testing of firewalls as well as input validation mechanisms.

Offutt et al. introduced the concept of Bypass Testing in which an application's input validation is tested for robustness and security [38]. Tests are generated to intentionally violate client-side input checks and are then sent to the server application to test whether the input constraints are adequately evaluated. Liu et al. [35] proposed an automated approach to recover an input validation model from program source code and formulated two coverage criteria for testing input validation based on the model. Desmet et al. 17] verify a given combination of a WAF and a web application for broken access control vulnerabilities, e.g. forceful browsing, by explicitly specifying the interactions of application components on the source code level and by applying static and dynamic verification to enforce only legal state transitions. In contrast, we propose a blackbox technique that does not require access to source code or client-side input checks to generate test cases. In our approach, we use machine learning to identify the patterns recognized by the firewall as SQLi attacks and generate bypassing test cases that avoid those patterns.

Tripp et al. [48] proposed XSS Analyzer, a learning approach to web security testing. The authors tackle the problem of efficiently selecting from a comprehensive input space of attacks by learning from previous attack executions. Based on the performed learning, the selection of new attacks is adjusted to select attacks with a high probability of revealing a vulnerability. More specifically, XSS Analyzer generates attacks from a grammar and learns constraints that express which literals an attack cannot contain in order to evade detection. The authors find that XSS Analyzer outperforms a comparable state-of-the-art algorithm, thus, suggesting that learning input constraints (a concept similar to the path conditions in ML-Driven) is effective to guide the test case generation.

In contrast to our work, XSS Analyzer applies learning to individual literals only while our approach also learns if a combination of literals is likely to bypass or be blocked. Therefore, XSS Analyzer cannot capture more complex input constraints involving multiple literals simultaneously, e.g., an attack should contain literal $a$, but not $b$ and $c$ in order to evade detection. Furthermore, to analyze which literals in an attack are blocked, XSS Analyzer first splits each attack into its composing tokens; then, it resends each token to the target web application. Since multiple attacks can share the same tokens, XSS Analyzer sends each token only once to avoid performing the same analysis multiple times. This procedure consumes a large quantity of HTTP requests. In contrast, ML-Driven does not require to resubmit individual slices, but learns path conditions solely from previously executed test case and, thus, spends its test budget more efficiently. In addition, there are differences between XSS Analyser and ML-Driven in terms of objectives: The former addresses cross-site scripting sanitization in web applications, while the latter addresses the detection of SQLi attacks in WAFs.

In grammar-based testing, a strategy typically samples a large input space defined by a grammar. Several grammarbased approaches exist in the literature for testing security properties of an application under test [37], [47]. Godefroid et al. [23] proposed white-box fuzzing, which starts by executing an application under test with a given wellformed input and uses symbolic execution to create input constraints when conditional statements are encountered on the execution path. Then, new inputs are created that exercise different execution paths by negating the previously collected constraints. The implementation of the approach found a critical memory corruption vulnerability in a fileprocessing application. In a follow-up work, the authors propose grammar-based white-box fuzzing [21], which addresses the generation of highly structured program inputs. In this work, well-formed inputs are generated from a grammar and the constraints created during execution are expressed as constraints on grammar tokens. To generate new inputs, a constraint solver searches the grammar for inputs that satisfy the constraints. The authors implemented their work in a tool named $S A G E$ and found several securityrelated bugs [22]. In contrast to the mentioned work of Godefroid et al., our work does not require access to the source code of the application under test, which is in many 
practical scenarios not available, but proposes a black-box approach based on machine learning to efficiently sample the input space defined by an attack grammar.

The topic of testing network firewalls has also been addressed by an abundant literature. Although network firewalls operate on a lower layer than application firewalls, which are our focus, they share some commonalities. Both use policies to decide which traffic is allowed to pass or should be rejected. Therefore, testing approaches to find flaws in network firewall policies might also be applicable to web application firewall policies. Bruckner et al. [1] proposed a model-based testing approach which transforms a firewall policy into a normal form. Based on case studies they found that this policy transformation increases the efficiency of test case generation by at least two orders of magnitude. Hwang et al. |29| defined structural coverage criteria of policies under test and developed a test generation technique based on constraint solving that tries to maximize structural coverage. Other research has focused on testing the firewalls implementation instead of policies. Al-Shaer et al. [3] developed a framework to automatically test if a policy is correctly enforced by a firewall. Therefore, the framework generates a set of policies as well as test traffic and checks whether the firewall handles the generated traffic correctly according to the generated policy. Some authors have proposed specification-based firewall testing. Jürjens et al. [30] proposed to formally model the tested firewall and to automatically derive test cases from the formal specification. Senn et al. [43] proposed a formal language for specifying security policies and automatically generate test cases from formal policies to test the firewall. In contrast, in addition to targeting application firewalls, our approach does not rely in any models of security policies or the firewall under test, such formal models are rarely available in practice.

\section{APPROACH}

This section introduces an approach for testing WAFs. Section 3.1 defines the input space for this testing problem as a context-free grammar. Section 3.2 presents a simple attack generation strategy that randomly samples the input space and serves as baseline. Section 3.3 presents two test generation strategies that make use of machine learning to guide test generation towards areas in the input space that are more likely to contain successful attacks. Finally, Section 3.4 details an approach to combine such attack generation strategies to achieve better results.

\subsection{A Context-Free Grammar for SQLi Attacks}

SQLi attacks (or test cases in this context) are small "programs" that aim at changing the intent of the target SQL queries they are injected in. We systematically surveyed known SQLi attacks published in the literature, e.g., [5], [6], 24 and from other sources e.g., OWASI ${ }^{2}$ and SqlMap Then, we defined a context-free grammar for generating and analyzing SQLi attacks.

\footnotetext{
2. https://www.owasp.org

3. http://sqlmap.org
}

We consider three main categories of SQLi attacks in our grammar: (i) Boolean, (ii) Union, and (iii) Piggy-Backed. These type of attacks aim at manipulating the intended logic by injecting additional SQL code fragments in the original SQL queries. We briefly discuss each attack category and provide example attacks that can be derived using our grammar. For a detailed discussion refer to the literature [10], [27].

Boolean Attacks. The intent of a boolean SQLi attack is to influence the where clause within a SQL statement to always evaluate either to true or false. As a result, a statement, into which a boolean SQLi attack is injected, returns on its execution either all data records of the queried database tables (in case the where clause evaluates always to true) or none (in case the where clause evaluates always to false). This attack method is typically used to bypass authentication mechanisms, extract data without authorization, or to identify injectable parameters. The example described in Section 2.1 is an instance of boolean attacks.

Union Attacks. The union keyword joins the result set of multiple select statements and, hence, union $S Q L i$ attacks are typically used to extract data located in other database tables than the original statement is querying. For example, consider an application that retrieves a list of product names based on a search term. The SQL statement to retrieve the product names might be:

$$
\begin{gathered}
\text { SELECT name FROM products WHERE name LIKE } \\
\text { "\%search term\%" }
\end{gathered}
$$

where search term is a string provided by the user. If the SQL statement is formed in an insecure way, an attacker could provide the search term phone " UNION SELECT passwd FROM users \#", which would result in the statement:

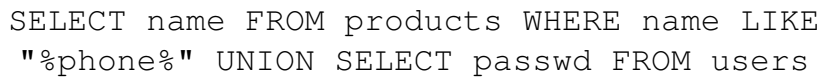

Hence, in addition to a list of products containing the search term phone, the attacker could obtain the passwords of all users with the modified query above.

Piggy-Backed Attacks. In SQL, the semicolon (;) can be used to separate two SQL statements. Piggy-Backed attacks use the semicolon to append an additional statement to the original statement and can be used for a wide range of attack purposes (e.g. data extracting or modification, and denial of service). An example of a piggy-backed attack is ; DROP TABLE users \#. If this attack is injected into a vulnerable SQL statement, it drops the table user and, thus, potentially breaks the application.

The grammar. We defined a grammar for SQLi attacks in the Extended Backus Normal Form, which is publicly available ${ }^{4}$ on GitHub. An excerpt of the grammar is depicted in Figure 1. The start symbol of the grammar is $\langle$ start $\rangle$, while "::=" denotes the production symbol, "," is concatenation, and "|" represents alternatives (grammar rule 1 of Figure 1). The grammar covers three different contexts in which SQLi attacks can be inserted into: numericCtx, sQuoteCtx, and dQuoteCtx. SQLi attacks belonging to the first context yield syntactically correct statements if they are injected in a numerical context (rule 2 of Figure 1 . For

4. https://github.com/dappelt/xavier-grammar 
example, the attack 1 OR true belongs to numeric $C t x$ and yields a syntactically correct statement if injected into the statement SELECT * FROM person WHERE age $=<$ user input $>$ where the placeholder $<$ user_input $>$ is intended to be replaced with a numerical value. Similarly, the second and third context, sQuoteCtx and dQuoteCtx, target SQL statements in which the user input is used as string literal and surrounded by single quotes (rule 3 of Figure 1 and double quotes (rule 4), respectively. For example, the SQLi attack " OR "a"="a belongs to dQuoteCtx and yields a syntactically correct statement if injected into SELECT * FROM person where name $="<$ user_input $>$ ".

Our grammar can be extended to incorporate other variants of SQLi attacks. For example, the non-terminal $\langle$ blank $\rangle$ (rule 30 of Figure 1) can have more semantically equivalent terminal characters:,$+ /^{* *} /$, or unicode encodings: $\% 20$, $\% 09, \% 0 a, \% 0 b, \% 0 c, \% 0 d$ and $\% a 0$; the quotes (single or double) can be represented using HTML encoding, and so on. Since the grammar is an input to our approach it can also be replaced with alternative grammars, which define an input space for different attack types (e.g. cross-site scripting or XML injection).

\subsection{Grammar-based Random Attack Generation}

Based on the proposed grammar, the random attack generation (RAN) procedure is straightforward: Beginning from the start symbol $\langle$ start $\rangle$, a randomly selected production rule is applied recursively until only terminals are left. Since there is no loop in the grammar, this attack generation procedure will always terminate. The output SQLi attack is produced by concatenating all terminal symbols appearing in the generated instance of the grammar.

To produce a set of diverse random SQLi attacks that yields a good coverage of the grammar, each production rule is selected with a probability proportional to the number of distinct production rules descending from the current one.

Among the techniques presented in this work, RAN implements the simplest strategy for sampling the input space defined by the attack grammar. Indeed, multiple SQLi attacks can be generated by simply re-applying RAN multiple times until the maximum number of tests/attacks (or the maximum running time) is reached. Bypassing attacks are then detected by executing all generated SQLi attacks against the target WAF.

\subsection{Machine Learning-Guided Attack Generation}

As the difficulty to find bypassing attacks increases, i.e. a WAF detects a large proportion of attacks, a random attack generation strategy (e.g., RAN) will increasingly become inefficient. In such situations, a more advanced test generation strategy that spends more computational effort to identify test cases with a higher bypassing probability is expected to be more efficient. In this section, we introduce a machine learning-based approach, called ML-Driven, to sample the input space in a more efficient manner than RAN does.

Theoretically, SQLi attacks could be generated using search-based testing techniques [4], [12], [36], [40]. However, the application of these techniques requires the definition of a distance function $f$ that measures the distance between a coverage target to reach and the execution results of a
1. $\langle$ start $\rangle::=\langle$ numericCtx $\rangle \mid\langle$ sQuoteCtx $\rangle \mid\langle\mathrm{dQuoteCtx}\rangle$;

Injection Context

2. $\langle$ numeric $C t x\rangle::=\langle$ digitZero $\rangle,\langle$ wsp $\rangle,\langle$ booleanAtk $\rangle,\langle$ wsp $\rangle$

I $\langle$ digitZero $\rangle,\langle$ parC $\rangle,\langle w s p\rangle,\langle$ booleanAtk $\rangle,\langle w s p\rangle,\langle$ operOr $\rangle$, $\langle$ parO $\rangle$, 〈digitZero $\rangle$

I 〈digitZero $\rangle,[\langle$ parC $\rangle],\langle$ wsp $\rangle,\langle$ sqliAtk $\rangle,\langle$ comment $\rangle$;

3. $\langle s Q$ uoteCtx $\rangle::=\langle$ squote $\rangle,\langle$ wsp $\rangle,\langle$ booleanAtk $\rangle,\langle$ wsp $\rangle,\langle$ operOr $\rangle$, $\langle$ squote $\rangle$

I $\langle$ squote $\rangle,\langle$ parC $\rangle,\langle w s p\rangle,\langle$ booleanAtk $\rangle,\langle w s p\rangle,\langle$ operOr $\rangle$, $\langle$ parO $\rangle,\langle$ squote $\rangle$

I $\langle$ squote $\rangle,[\langle$ parC $\rangle],\langle$ wsp $\rangle,\langle$ sqliAtk $\rangle,\langle$ comment $\rangle$;

4. $\langle$ dQuoteCtx $\rangle::=\langle$ dquote $\rangle,\langle$ wsp $\rangle,\langle$ booleanAtk $\rangle,\langle$ wsp $\rangle,\langle$ operOr $\rangle$, $\langle$ dquote $\rangle$

। $\langle$ dquote $\rangle,\langle$ parC $\rangle,\langle$ wsp $\rangle,\langle$ booleanAtk $\rangle,\langle w s p\rangle,\langle$ operOr $\rangle$, $\langle$ parO $\rangle,\langle$ dquote $\rangle$

I $\langle$ dquote $\rangle,[\langle$ parC $\rangle],\langle$ wsp $\rangle,\langle$ sqliAtk $\rangle,\langle$ comment $\rangle$;

5. $\langle$ sqliAtk $:::=\langle$ unionAtk $\rangle$ | $\langle$ piggyAtk $\rangle|\langle$ booleanAtk $\rangle$;

Union Attacks

6. $\langle$ unionAtk $\rangle:=\langle$ union $\rangle,\langle$ wsp $\rangle,[\langle$ unionPostfix $\rangle],\langle$ operSel $\rangle,\langle w s p\rangle$ $\langle$ cols $\rangle$

I $\langle$ union $\rangle,\langle$ wsp $\rangle,[\langle$ unionPostfix $\rangle],\langle$ parO $\rangle,\langle$ operSel $\rangle,\langle$ wsp $\rangle$, $\langle$ cols $\rangle,\langle$ parC $\rangle$;

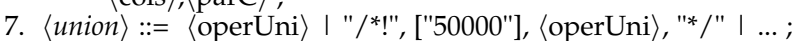

8. $\langle$ unionPostfix $\rangle::=$ "all", $\langle w s p\rangle \mid$ "distinct", $\langle w s p\rangle$;

Piggy-backed Attacks

9. $\langle$ piggyAtk $\rangle::=\langle$ operSem $\rangle,\langle$ operSel $\rangle,\langle$ wsp $\rangle,\langle$ funcSleep $\rangle \mid \ldots$;

Boolean-based Attacks

10. $\langle$ booleanAtk $\rangle::=\langle$ orAtk $\rangle \mid\langle$ andAtk $\rangle$;

11. $\langle$ orAtk $\rangle::=\langle$ operOr $\rangle,\langle$ booleanTrueExpression $\rangle$;

12. $\langle$ and Atk $\rangle::=\langle$ operAnd $\rangle,\langle$ booleanFalseExpression $\rangle$;

13. 〈booleanTrueExpression $\rangle::=\langle$ unaryTrue $\rangle \mid\langle$ binaryTrue $\rangle$;

SQL Operators and Keyword

15. $\langle$ operNot $\rangle::=$ "!" | "not" ;

16. 〈operBinInvert $\rangle::="$ ";

17. $\langle$ operEqual $\rangle::="="$;

18. $\langle$ operLt $\rangle::="<"$;

19. $\langle$ operGt $\rangle::=">"$;

20. $\langle$ operLike $\rangle::=$ "like" ;

21. 〈operIs $\rangle::=$ "is" ;

22. 〈operMinus $\rangle::="-"$;

23. $\langle$ operOr $\rangle::=$ "or" | "| |" ;

24. $\langle$ operAnd $\rangle::=$ "and" | "\&\&" .

25. $\langle$ operSel $\rangle::=$ "select"

26. 〈operUni $::=$ "union" ;

27. $\langle$ operSem $\rangle::=" ; "$;

28. 〈comment $::=$ "\#" | $\langle$ ddash $\rangle,\langle$ blank $\rangle$;

29. $\langle$ ddash $\rangle::="--"$

\section{Obfuscation}

30. $\langle$ inlineComment $\rangle::=" / * * / "$;

31. $\langle$ blank $\rangle::="$ ";

32. $\langle$ ws $p\rangle::=\langle$ blank $\rangle$ | $\langle$ inlineComment $\rangle$

Fig. 1. Excerpt of the grammar used in the paper expressed in Extended Backus Normal Form.

given test case [36], [39], [46]. For example, in white-box unit testing, approach level [36] and branch distance [36] are used to determine how far is the execution path of test $t$ from covering a given branch in the control flow graph. However, in the context of security testing, the target SQLi vulnerabilities are not known a-priori and, thus, such a distance function $f$ cannot be defined. Therefore, we face the problem to efficiently choose from a large set of SQLi attacks the ones that are more likely to reveal holes in the WAF under test. The problem is challenging because there is little information available to estimate how close a test comes to 
bypassing the WAF. When a test is executed, only one of the following two events can be observed: bypassing, or blocked. This leaves the search with no guidance to effectively assess how close a blocked attack is from bypassing the WAF. Lack of guidance is a well-known issue to address when applying search-based techniques since previous studies (e.g., [44]) showed that sophisticated search algorithms (e.g., evolutionary algorithm) do not provide any advantage over random search (i.e., the approach in Section 3.2) when the gradient of the function $f$ has many plateaus (flag problem).

To tackle this problem, we introduce ML-Driven, a search-based technique that uses machine learning to model how the elements (attributes of attacks) of the tests are associated with the likelihood of bypassing the WAF, the latter being used to guide the search. In the search process, tests that are predicted to have such high likelihood are considered to have a high fitness and are more likely to be generated. ML-Driven first employs random test generation, as described in the previous section, to generate an initial training set. Then, these tests are sent to a web application protected by the WAF, and are labelled as " $\mathrm{P}$ " or " $\mathrm{B}$ " depending on whether they bypass or are blocked by the WAF, respectively. Tests and execution results are then encoded and used as initial training data to learn a model estimating the likelihood $(f)$ with which tests can bypass the WAF. Using this measure we can rank, select, and modify tests associated with high $f$ values to produce new tests. These new tests are then executed, and their results (" $\mathrm{P}$ " or " $\mathrm{B}$ ") are used to improve the prediction model, which will in turn help generating more distinct tests that bypass the WAF.

In what follows, we will discuss in detail the procedure used to decompose and encode test cases into a training set, and the machine learning algorithms that are used to estimate the likelihood of each test to bypass the WAF. Finally, we describe ML-Driven, our search-based approach guided by machine learning.

\subsubsection{Attack Decomposition}

We can derive a test from the grammar by applying recursively its production rules. This procedure can be represented as a derivation tree. A derivation tree (also called parse tree) of a test is a graphical representation of the derivation steps that are involved in producing the test. In a derivation tree, an intermediate node represents a non-terminal symbol, a leaf node represents a terminal symbol, and an edge represents the applied production. Fig. 2 depicts the derivation tree of the boolean attack: , $O R " a "=" a " \#$. In the course of generating this test, we first apply the $\langle$ start $\rangle$ rule:

$$
\begin{aligned}
& \langle\text { start }\rangle \quad::=\langle\text { numericCtx }\rangle \mid\langle\text { sQuoteCtx }\rangle \\
& \text { I 〈dQuoteCtx>; }
\end{aligned}
$$

and derive $\langle$ Q QuoteCtx $\rangle$. We then apply the third rule of the grammar to derive $\langle s q u o t e\rangle,\langle w s p\rangle$, $\langle$ sqliAtk $\rangle$, and $\langle$ comment $\rangle$. This procedure is repeated until all non-terminal symbols are transformed into terminal symbols. The attack string represented by a derivation tree is obtained by concatenating the leaves from left to right.

We use derivation trees to identify which substrings of a SQLi attack are likely to be responsible for the attack being blocked or not. Specifically, an attack is divided into

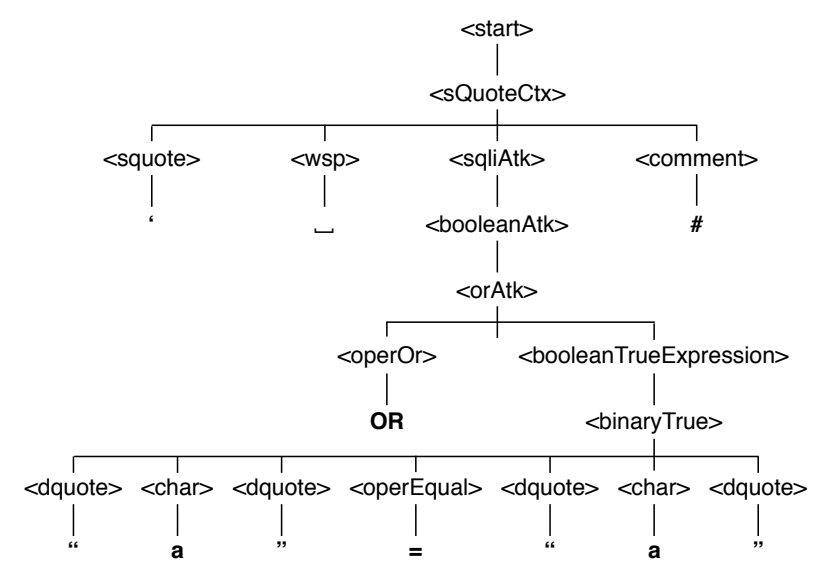

Fig. 2. The derivation tree of the "boolean" SQLi attack: '

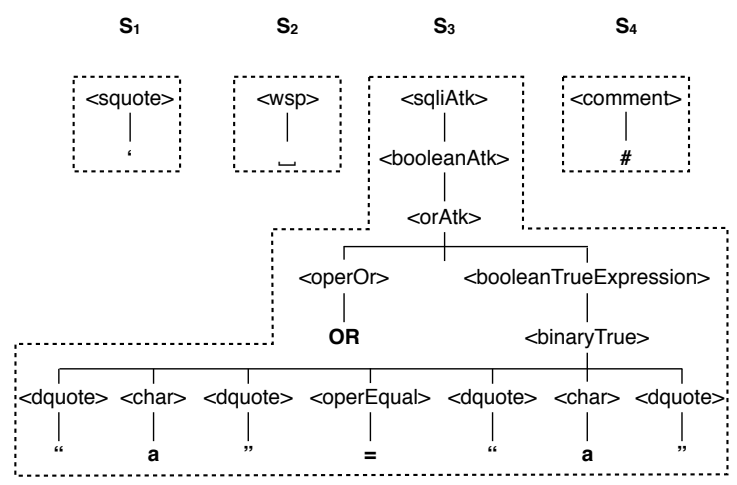

Fig. 3. Example subset of slices decomposed from the tree in Fig. 2

substrings by decomposing its derivation tree into slices. The definition of a slice is as follows:

Definition 1 (Slice). A slice $s$ is a subtree $T^{\prime}$ of a derivation tree $T$ such that $T^{\prime}$ contains a strict subset of leaves of $T$.

A slice is supposed to represent a substring of an attack, hence only subtrees of a derivation tree that contain a subset of leaves are considered to be slices. Otherwise, if the subtree contains the same leaves as the derivation tree, the represented string is not a substring, but the same string as the derivation tree. For example, for the derivation tree in Fig. 2 the subtree with the root $\langle s Q u o t e C t x\rangle$ contains all the leaves of the whole derivation tree and, therefore, is not a slice. A sample of four slices decomposed from the derivation tree of this example is depicted in Fig. 3 Among all possible slices of a derivation tree, we are interested in extracting the minimal ones, i.e., slices that cannot be further divided into sub-slices:

Definition 2 (Minimal Slice). A slice $s$ is minimal if it has only two nodes: a root and only one child that is a leaf.

The procedure to decompose a derivation tree into slices is detailed in Algorithm 1. Starting from the root node the algorithm recursively computes slices from descendant nodes by calling VISIT(child, root, $S$ ) in line 5 . In line 11, the condition (root.leafs $\backslash$ node.leafs) $\neq \emptyset$ ensures that only subtrees complying with Def. 1 are considered. In line 14, the recursion ends if the node forms a minimal slice, as defined in Def. 2, otherwise the recursion continues. 


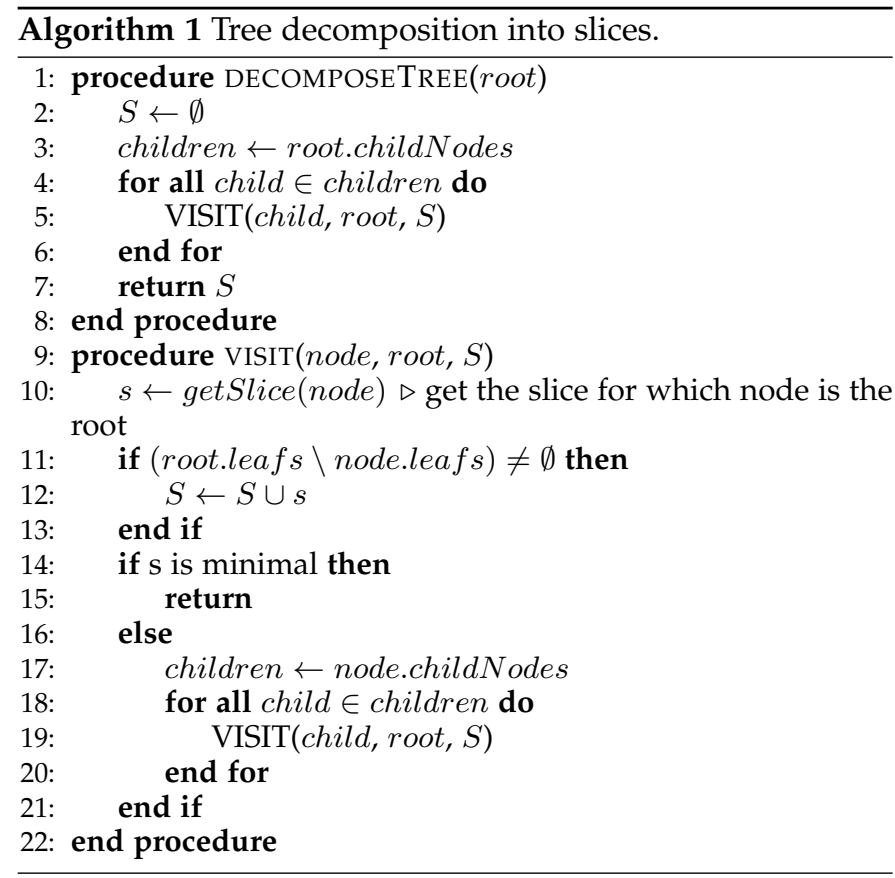

For example, applying the decomposition procedure to the derivation tree in Fig. 2 yields a set of 12 distinct slices.

We conjecture that the appearance of one or more slices in a test could result in the test getting blocked or not. In the next sections, we develop this idea further by analyzing slices of a collection of tests and predicting, using machine learning, how their appearance in the tests affect their likelihood of bypassing a WAF or being blocked.

\subsubsection{Training Set Preparation}

Given a set of tests that have been labelled with their execution result against a WAF, that is a " $\mathrm{P}$ " or " $\mathrm{B}$ " label, we transform each test into an observation instance to feed our machine learning algorithm.

1) Each test is decomposed into a vector of slices $t_{i}=$ $\left\langle s_{1}, s_{2}, \ldots, s_{N_{i}}\right\rangle$ by applying the attack decomposition procedure.

2) Each slice is assigned a globally unique identifier. If the same slice is part of multiple tests, it is referenced by the same identifier. We map each unique slice to an attribute (a feature) of the training data set for machine learning.

3) Every test is transformed into an observation of the training data set by checking whether the slices used as attributes are present or not in the corresponding vector of slices of the test.

As a concrete example, let us consider three tests $t_{1}, t_{2}, t_{3}$; the first two are blocked while the last can bypass a WAF. Their decompositions into slices and labels are shown on the left side of Table 1, and their encoded representation on the right side of the table. In total, we have five unique slices from all the tests and they become attributes of the training data for machine learning. If a slice appears in a test, its corresponding attribute value in the training data is " 1 ", and otherwise " 0 ".

\subsubsection{Decision Tree and Path Condition}

By decomposing tests into slices and transforming them into a labelled data set, we can now apply a supervised machine
TABLE 1

An example of test decompositions and their encoding.

\begin{tabular}{|c|c|c|c|c|c|c|c|c|c|}
\hline t.id & vector & label & t.id & $s_{1}$ & $s_{2}$ & $s_{3}$ & $s_{4}$ & $s_{5}$ & $\mathrm{clz}$ \\
\hline 1 & $\left\langle s_{1}, s_{2}, s_{3}\right\rangle$ & B & 1 & 1 & 1 & 1 & 0 & 0 & $\mathrm{~B}$ \\
\hline 2 & $\left\langle s_{1}, s_{2}, s_{4}\right\rangle$ & B & 2 & 1 & 1 & 0 & 1 & 0 & B \\
\hline 3 & $\left\langle s_{4}, s_{5}\right\rangle$ & $\mathrm{P}$ & 3 & 0 & 0 & 0 & 1 & 1 & $\mathrm{P}$ \\
\hline
\end{tabular}

learning technique to predict which slices or combinations of slices are associated with tests bypassing or being blocked by the WAF. To be able to identify such slices, we rely on machine learning techniques that provide an interpretable output model. That is, the reason for the classification of attacks into bypassing or blocked should be easily comprehensible. Therefore, though other algorithms generating classification rules could have been used as well, we selected decision trees (DTs) for this task.

A DT is a tree structure with decision nodes and leaves; a decision node represents an attribute from a data set; each branch from such a node represents a possible value for the corresponding attribute; and a leaf node represents a classification for all instances that reach this node. In our context, each decision node represents a slice and the branches from the node can be " 0 " or " 1 ", corresponding to whether the slice is absent or present. A leaf node classifies instances into blocked or bypassing and is labelled accordingly with "B" or " $\mathrm{P}$ ". Fig. 4 shows an example decision tree learned from the data in Table 1

The paths from the root node of the decision tree to its leaf nodes embody the combinations of slices which are likely to be the reason for tests to bypass or to be blocked, depending on the classification. More generally, we define a concept of path condition as:

Definition 3 (Path Condition). A path condition represents a set of slices that the machine learning technique deems to be relevant for the attack's classification into blocked or bypassing. The path condition is represented as a conjunction $\bigwedge_{i}^{k}\left(s_{i}=v a l\right)$, in which val $=1 \mid 0$, and $k$ is the number of relevant slices.

The procedure for computing path conditions depends on the machine learning algorithm that is used to build decision trees. We have selected two alternative algorithms and assessed their overall impact on test results.

RandomTree. The most prominent difference to classical decision tree algorithms (e.g. C4.5 [42]) is that RandomTree relies on randomization for building the decision tree [15]. When selecting an attribute for a tree node, the algorithm chooses the best attribute amongst a randomly selected subset of attributes. By choosing only subset of attributes, the algorithm scales well with the size of the training data set. In our context, a scalable learner is important since the data sets contain a larger number of attributes and the decision tree is frequently rebuilt, as described in Section 3.3.4

Given a decision tree and a slice vector $V$ of a test $t$, we can obtain the path condition for $t$ by visiting the decision tree from the root and check the presence (value = 1 ) or absence (value $=0$ ) of the attributes encountered with respect to $V$. The procedure stops once a leaf is reached. For instance, Fig. 4 presents a decision tree learned from the example data discussed in Table 1 . For test $t_{1}$, the attribute $s_{3}$ is present in the test's slice vector $\left\langle s_{1}, s_{2}, s_{3}\right\rangle$, and thus $t_{1}$ follows the left branch. Hence, the path condition is 


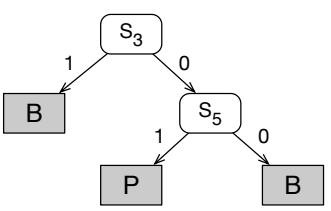

Fig. 4. An example of a decision tree obtained from the training data in Table 1

$\left(s_{3}=1\right)$. Similarly, for test $t_{3}$ with the slice vector $\left\langle s_{4}, s_{5}\right\rangle$, the attribute $s_{3}$ is not present, thus the right branch is followed leading to attribute $s_{5}$, which is present in the slice vector. Therefore, the resulting path condition for $t_{3}$ is $\left(s_{3}=0 \wedge s_{5}=1\right)$.

RandomForest. Machine classification methods are wellknown to be unstable, i.e., a small change in the training data can result in a different classification model [51]. Ensemble methods have been proposed to address the issue. In essence, multiple models are learned so that their collective predictions can mitigate bias in individual models. In this work, we implement an ensemble of classifiers to guide the test generation, i.e., instead of using only one RandomTree, we extend our technique to make use of ensembles of trees produced by RandomForest [15]. However, the benefits of RandomForest come at the cost of an increased computational overhead, e.g. learning an ensemble of RandomTrees takes longer than learning only a single RandomTree. We evaluate in Section 5 whether the benefits justify the increased overhead.

A RandomForest consists of multiple RandomTrees. To classify an attack with RandomForest, each individual RandomTree first classifies the attack and computes the prediction confidence (an estimated probability for the classification is to be correct). Then, all individual classifications are consolidated by computing the average of the prediction confidence values for each class. Finally, the class with the highest prediction confidence is chosen by RandomForest as final classification.

To compute the path condition for a given attack with RandomForest, for all trees that classify the attack as bypassing a path condition is computed separately. Thereby, the path condition for each tree is computed according to the previously described procedure. The overall path condition for the entire RandomForest is, then, the conjunction of the path conditions computed from the trees.

\subsubsection{ML-Driven Evolutionary Testing Strategy}

To increase the likelihood of generating bypassing attacks, we propose a ML-driven evolutionary testing strategy whose pseudo-code is detailed in Algorithm 2 It combines machine learning algorithms (either RandomTree or RandomForest) with the classical $(\mu+\lambda)$ Evolutionary Algorithm (EA), which is a population-based evolutionary algorithm with a population size $\mu$ and a restricted number of $\lambda$ offsprings.

As any EA, Algorithm 2 starts with an initial set of solutions (initTests) usually called population. The initial population can be either generated by the random attack generator from Section 3.2 or by selecting tests from existing test suites. The initial tests are executed against a target WAF (line 2) if their execution results are not yet known (i.e., in case of randomly generated tests). Each solution is assigned a fitness score according to its probability of

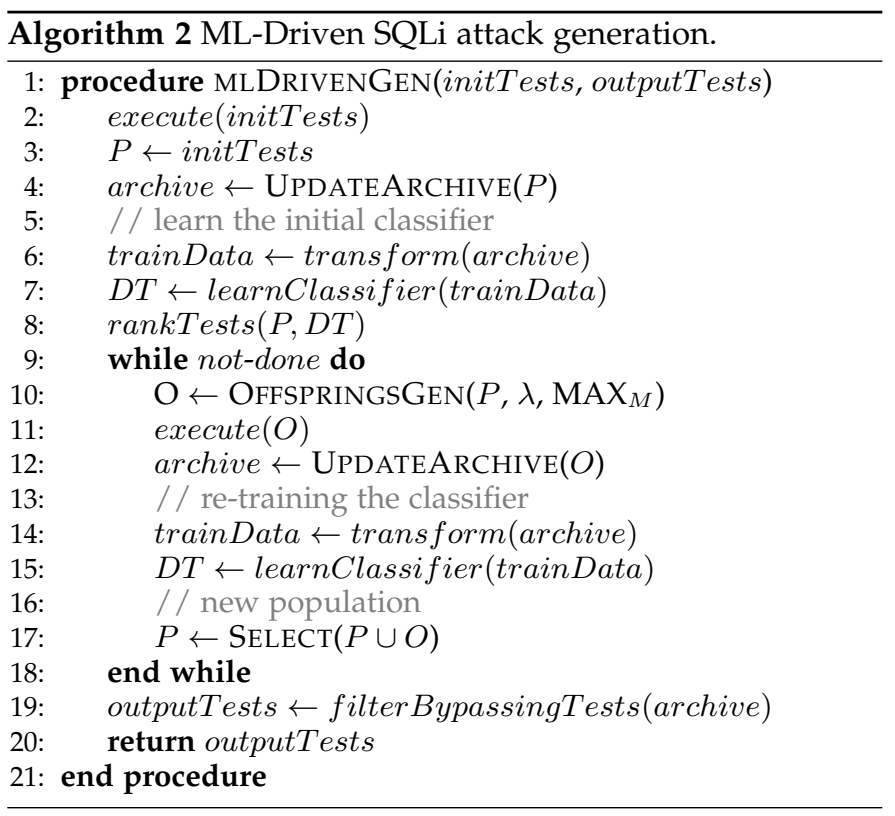

bypassing the WAF determined the classifier built in lines 5 6 of Algorithm 2 The classifier is built as follows: (i) the tests are transformed into the training set (routine trainData in line 6); (ii) a classifier $D T$ is then trained from the data (line 7 of Algorithm 2). Then, individuals in the initial population are ranked using the $D T$ classifier (routine rankTests in line 8), which assigns each individual (test) a probability of bypassing the WAF.

In the main loop in lines 9-18, the population is evolved through subsequent iterations, called generations. $\lambda$ new solutions are generated using the routine OFFSPRINGSGEN in line 10. Such a routine selects the best individuals (parents) and mutates them to generate $\lambda$ new individuals, called offsprings. The offsprings are executed against the target WAF (line 11) and labelled as "bypassing" or "blocked" depending on their execution results. In line 12, the newly generated tests are added to an archive (line 12), which is a second population used to keep track of all tests being generated across the generations [39]. Then, the $D T$ classifier is retrained by converting the archive into the training set (line 14) and re-applying the ML training (line 15). At the end of each generation, a new population is formed in line 17 by selecting the fittest $\mu$ tests among parents and offsprings according the $D T$ classifier. The loop terminates when the maximum number of generations (or the maximum running time) is reached (condition not-done in line 9).

Therefore, the three key components of Algorithm 2 are: (i) the ML-driven fitness assignment; (ii) the selection operator; and (iii) the mutation operator applied for generating offsprings. These key components are detailed in the following paragraphs.

ML-driven fitness assignment. In this paper, each candidate test $t$ is assigned a fitness score by using machine learning algorithms. In Algorithm 2 a classifier DT is built at the beginning of the search (lines 6-7) at the end of each generation (lines 14-15 of Algorithm 2) using the archive as training set. Such an archive is updated whenever new test cases (either "bypassing" or "blocked") are generated (lines 4,12 ). To build the classifier, the archive is transformed 


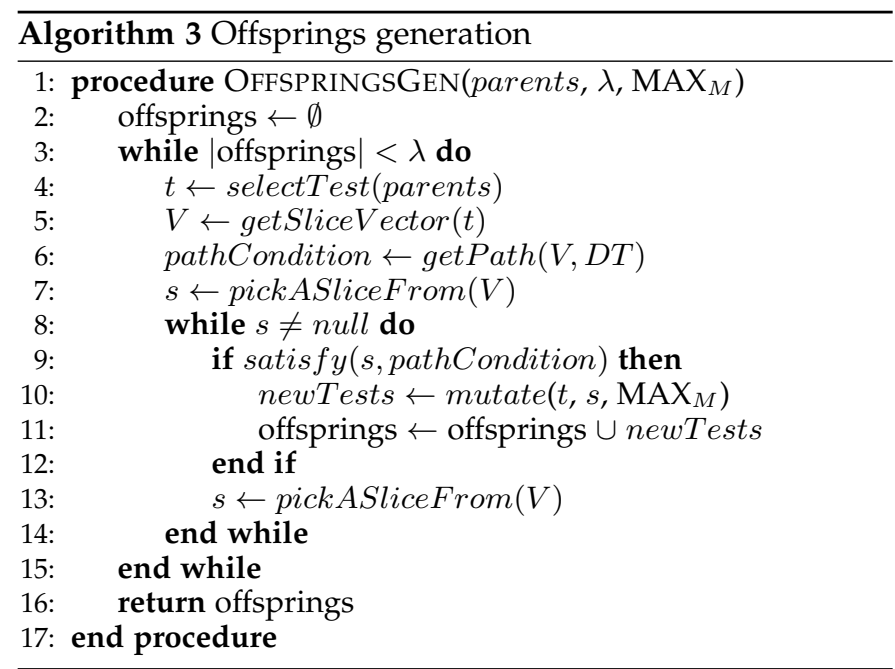

into a training set (lines 6,7) using the steps discussed in Section 3.3.2. Then, the classifier $D T$ is trained using either RandomTree or RandomForest. Once built, DT can be used to estimate the probability of a given test $t$ to bypass the target WAF depending on which slices appear in the three decomposition of $t$ (see Section 3.3.1).

Elitist selection. In $(\mu+\lambda)$-EAs, $\mu$ parents and $\lambda$ offsprings compete to form the new population of $\mu$ individuals for the next generation. In Algorithm 2, this procedure is implemented by the routine SELECT (line 17). Once the fitness assignment has been performed using $D T$, the tests in the current population are ranked in descending order of their bypassing probability. The top $\mu$ test cases in the ranking are selected to form the next population.

Generating offsprings. The routine used to generate offsprings is detailed in Algorithm 3 Given a set of $\mu$ parents, such a routine generates mutants from each parent until reaching a total number of $\lambda$ offsprings (loop condition in line 3 of Algorithm 3). In each iteration of the loop in lines 3-15, Algorithm 3 selects the test with the highest rank as candidate test for mutation. If more than one candidate have tied ranks, the selection is random among them. If a test has been selected before, it will not be selected again. For each selected parent $t$ (line 4 ), offsprings are generated starting from its corresponding path condition (pathCondition), which is determined using the routine getPath (line 6) from the slice vector $V$ of the attack $t$ (obtained in line 5).

Then, offsprings are generated from a parent $t$ by replacing its slices $s$ in $V$ with other alternative slices according to our grammar. In particular, one slice $s$ is randomly picked from the slice vector $V$ of the attack $t$ (line 7) and it is replaced with an alternative slice $s^{\prime}$ to generate new tests (routine mutate in line 10). Both $s$ and $s^{\prime}$ have to satisfy the given path condition. That is, they either appear in the predicate and comply with it, or do not appear in the predicate of the path condition. If $s$ does not satisfy the path condition, it is not considered for generating mutants/offsprings (condition in line 9).

To better explain this mutation procedure, let us consider the test $t_{2}$ from Table 1 with slice vector $\left\langle s_{1}, s_{2}, s_{4}\right\rangle$. According to the slices in Figure 3 , the path condition for $t_{2}$ is $\left(s_{1}=1\right)$; thus, we can select $s_{2}$ or $s_{4}$ and replace them with their alternatives. Equivalent alternatives of a slice are determined based on the root symbol of the slice and all production rules of the grammar that start with this symbol. For example, taking slice $s_{2}$ in Fig. 3 that starts with $\langle w s p\rangle$ and derives $\langle$ blank $\rangle$, we obtain only one production rule from the grammar:

$$
\langle\text { wsp }\rangle::=\langle\text { blank }\rangle \mid\langle\text { inlineComment }\rangle \text {; }
$$

As a result, we determine only one alternative slice that starts with $\langle w s p\rangle$ and derives 〈inlineComment .

At the mutation step in line 10 of Algorithm 3, the parameter $\mathrm{MAX}_{M}$ is an integer value that limits the number of mutants that are generated for test $t$ and slice $s$. If the number of alternative slices for $t$ and $s$ is greater than $\operatorname{MAX}_{M}$ only $\mathrm{MAX}_{M}$ alternative slices are selected and used, in turn, to form mutants. If the number of alternative slices is lower than $M A X_{M}$, all available slices are used to form mutants. Therefore, at most $\mathrm{MAX}_{N}$ offsprings (mutants) are generated from each parent $t$, where each offspring differs from its parent in one single (alternative) slice.

Motivations. Let us now explain why we decided to opt for the classical $(\mu, \lambda)$-EA, which is a mutation-based evolutionary algorithm with no crossover operator. With this algorithm, $\mathrm{MAX}_{N}$ offsprings are generated from one single test $t$ via mutation only. Crossover, which is another well-know operator widely applied in various evolutionary algorithms (e.g., genetic algorithms), is not applied here. Usually, it generates two offsprings from a pair of solutions (parents) by randomly exchanging their genes. However, for our problem, a crossover operator cannot be defined since different solutions (tests) within the same population have different derivation trees that represent instances of incompatible derivation rules of our grammar. For example, let us assume we select for reproduction two test cases $t_{1}$ and $t_{2}$. The former instantiates the derivation rule $\langle$ start $\rangle$ $::=\langle$ numeric $C t x\rangle$ while the latter is an instance of the rule $\langle$ start $\rangle::=\langle s Q u o t e C t x\rangle$. If we apply any crossover operator (e.g., the single-point crossover), then the resulting two offsprings would violate our grammar since each of the new test case would contain slices from different (incompatible) derivation rules.

Exploration vs. Exploitation. Assuming that the total number of offsprings $(\lambda)$ to generate is constant, $\mathrm{MAX}_{M}$ controls how the evolutionary algorithm explores the test space either broadly (exploration) or deeply (exploitation). When $\operatorname{MAX}_{M}$ is small, the approach generates fewer offsprings per selected test, but selects more tests to mutate, thus exploring the test space in a broader fashion (higher exploration). When $\mathrm{MAX}_{M}$ is large, on the contrary, the approach generates more offsprings per selected test, but selects fewer tests to mutate, thus exploring the test space in a deeper fashion (higher exploitation). In the evaluation section two variants of ML-Driven are distinguished: ML-Driven B (broad, with $M A X_{M}=10$ ) and ML-Driven D (deep, with $M A X_{M}=100$ ). Section 3.4 presents a thorough analysis on how the parameter $M \overline{A X}_{M}$ influences the overall test results.

\subsection{Enhancing ML-Driven: An Adaptive Approach to Balance Exploration and Exploitation}

Maintaining a good balance between exploration and exploitation is extremely important for a successful application of EAs $|49|$. In our case, the balance is determined 
by the parameter $\operatorname{MAX}_{M}$, which affects the number mutants (offsprings) generated from each selected test. For ML-Driven D, $\mathrm{MAX}_{M}$ is set to a higher value, which leads selecting fewer tests for mutation $(\approx \lambda / 100)$, but generating more mutants per selected test (higher exploitation). For ML-Driven $\mathrm{B}, \mathrm{MAX}_{M}$ is set to a lower value, which leads selecting more tests for mutation $(\approx \lambda / 10)$, but generating fewer mutants per selected test (higher exploration). Notice that for both variants the total test budget is the same, but the allocation of the test budget differs.

A detailed analysis presented in Section 5.1. shows that no variant is superior over the other [8]: ML-Driven D performs better at the beginning of the search but ML-Driven $\mathrm{B}$ outperforms it in later stages. The reason for this phenomenon is because of the number of bypassing tests selected and their influence on the overall performance. At the beginning, there are only a few available tests with a high bypassing probability. Due to the higher value of $\mathrm{MAX}_{M}, \mathrm{ML}-\mathrm{Driven} \mathrm{D}$ is likely to select only these tests for mutation and, thus, exploits better the neighborhood of highly probable bypassing tests. This leads to generating more bypassing attacks in the earlier stages of the search. Reversely, since $\mathrm{MAX}_{M}$ is lower in ML-Driven B, more tests are selected for mutation, resulting in selecting not only the few tests with a high bypassing probability, but also tests with a low bypassing probability. As a result, ML-Driven $B$ generates fewer new bypassing tests.

On the other hand, after some iterations there are more tests with a high bypassing probability being available for mutation. In such a scenario, selecting more tests and mutating each one less often helps preserving diversity: ML-Driven B will explore the neighborhoods of multiple highly probable bypassing tests. ML-Driven D explores the neighborhoods of fewer tests while many other tests with similar bypassing probability remain unexplored.

In this section, we propose an improved variant of our attack generation strategy called ML-Driven E (Enhanced). Its goal is to combine the strengths of ML-Driven $B$ and ML-Driven D by adaptively adjusting the parameter $\mathrm{MAX}_{M}$ to better balance exploration and exploitation. We propose $M L-D r i v e n ~ E$, which is a more flexible approach for assigning the test budget to individual tests. Instead of generating a fixed number of mutants per test, as done with ML-Driven B/D, the number of mutants is calculated dynamically. The rationale of ML-Driven $E$ is twofold: First, the available test budget should be allocated only to tests with a high bypassing probability. Second, the test budget should be divided amongst all tests in proportion of that probability, thus favoring those more likely to yield new attacks.

Given the total number of offsprings $\lambda$ to generate, a set $T$ of tests selected as parents, the probability $P(t)$ of test $t$ to bypass the WAF, then the number of offsprings $m_{t}$ to generate for test $t$ is defined as:

$$
m_{t}=\frac{P(t)}{\sum_{x \in T} P(x)} * \lambda
$$

On the right-hand side, the fraction represents the relative bypassing probability of $t$ by dividing its bypassing probability with the sum of all bypassing probabilities of tests $x \in T$. By multiplying the relative bypassing probability

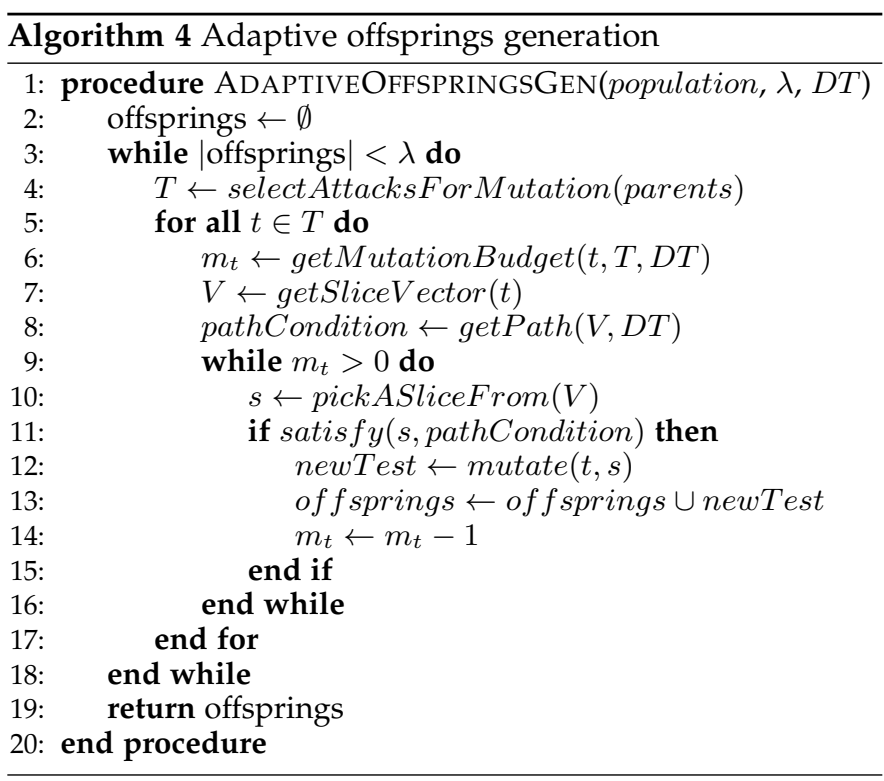

of test $t$ with $\lambda$ (total number of offsprings), $t$ is assigned a share of the total budget proportional to its relative bypassing probability. In other words, the number of offsprings/mutants to generate for each test $t$ is proportional to its bypassing probability in relation to the probabilities of all selected parents.

ML-Driven E shares the same pseudo-code with ML-Driven D and ML-Driven $B$ with the exception of the routine OFFSPRINGSGEN used to generate offsprings in Algorithm 2 Instead of using Algorithm 3. ML-Driven $E$ uses the new adaptive routine detailed in Algorithm 4 which includes the proposed modification to the budget calculation.

Similarly to Algorithm 3. Algorithm 4 generates $\lambda$ offsprings within the loop in lines 3-18. The differences concern (i) the number test cases selected as parents; and (ii) the number of mutants/offsprings generated from each individual parent. In line 4, the routine selectAttacksFor Mutation selects a set of attacks $T$ that have the highest bypassing probability from all available attacks in the current population. All attacks above a configurable threshold $\sigma$, e.g., in our experiment $\sigma=80 \%$, are selected. The loop from line 5 to 17 is executed for each selected attack in $T$. Within the loop, in line 6 the routine getMutationBudget calculates the number of offsprings/mutants $m_{t}$ to generate from attack $t$ with respect to $T$ and $D T$, the latter being the classifier. In other words, this method implements Equation 1. Lines 9 to 16 describe the mutation procedure for $t$, that is mostly unchanged from the original version of the algorithm, except that the number of generated offsprings/mutants for $t$ is set to $m_{t}$.

Since the number of tests with a bypassing probability larger than $80 \%$ may vary across generations, both the number of selected parents and the budget allocation vary over time. When there are only few tests with a large bypassing probability $(\geq \sigma)$, Algorithm 4 selects only those tests as parents. As result, the number of offsprings $m_{t}$ generated from each parent $t$ will be large (higher exploitation). Instead, when the current population contains many tests with a large bypassing probability $(\geq \sigma)$, the total budget 
of $\mu$ offsprings is shared among a larger set of parents. As a consequence, fewer mutants will be generated from each parent (higher exploration). Therefore, Algorithm 4 adaptively balances exploration and exploitation depending on the number tests in the current population that have a bypassing probability greater than $\sigma$.

\section{Empirical Study}

This section evaluates the proposed testing strategies in two separate case studies: a popular open-source WAF and a proprietary WAF that protects a financial institution. Section 4.1 introduces the case studies. Section 4.2 formulates the research questions. Section 4.3 explains the procedure we followed to execute the experiments while Section 4.5 describes the experiment variables.

\subsection{Subject Applications}

\subsubsection{Open-Source WAF}

In this case study, the firewall under test is ModSecurity, which implements the OWASP core rule set. ModSecurity is an open-source web application firewall that can be deployed with the Apache HTTP Server to protect web applications hosted under the server. Depending on the applications under protection, different firewall rule sets defined for different purposes can be used. The OWASP core rules target various kinds of attacks, e.g. Trojan, Denial of Service, and SQL Injection, and are maintained by an active community of security experts.

The web applications under protection are HotelRS, Cyclos, and SugarCRM. HotelRS is a service-oriented based system, providing web services for room reservation. It was developed and used in [16]. Cyclos is a popular open-source Java/Servlet Web Application for e-commerce and online payment $5^{5}$ SugarCRM is a popular customer relationship management system ${ }^{6}$. SugarCRM and Cyclos have been widely used in practice. In our experiment setting, the three applications are deployed on an Apache HTTP Server under Linux. ModSecurity is embedded within the web server; it protects the application's web services from SQLi attacks. Specifically, since these web services receive SOAP message ${ }^{7}$ from web clients, a malicious client can seed a SQLi attack string into a SOAP message and submit it to the web services in order to gain illegal access to data or functionality of the system.

In this paper, note that our testing target is the WAF that protects the applications, not the applications themselves, as our focus is on testing firewalls. HotelRS, SugarCRM, and Cyclos play solely the role of a destination for SQLi tests that bypass the WAF.

\subsubsection{Proprietary WAF}

In the industrial setting, we evaluate our approach on a proprietary WAF that is used in a corporate IT environment to protect back-end web services. These services are the backbone of a financial corporation and process thousands of transactions daily. To provide protection from malicious

5. http:/ / project.cyclos.org

6. http:// sourceforge.net

7. http://www.w3.org/TR/soap12-part1 requests, the WAF validates incoming requests in two steps: First, the values in a request are validated with respect to data types (e.g., string or numeric) and boundary constraints, e.g., a credit card number is expected to be a sequence of 16 to 19 digits. In a second step, each value is checked to make sure that it does not contain known malicious string patterns (i.e., using a SQLi blacklist) commonly used in attacks. Only if the request passes both validation steps the request is forwarded to the back-end services.

The computation time required to evaluate all testing strategies with the proprietary WAF is highly expensive because of (i) the slow responsive time of the web services, (ii) the number of testing strategies to compare, and (iii) the number of repetitions to perform for each testing strategy. Therefore, for the experiment we used of a highperformance cluster [2] when testing the proprietary WAF. Furthermore, we had to optimize a replica of the test environment to significantly decrease response times when invoking services.

In our optimized test environment, all configurations related to request filtering, that is whether a request is blocked or let through, are copied. Other configurations, e.g. logging or encryption, are disabled. The web application under protection is replaced by a simple mock-up application, which implements the same interface as the original application under protection. The mock-up replays a set of recorded responses from the original application and, thus, the WAF remains unaffected. Table 2 shows the message round trip time $(R T T)$ per operation computed over a time span of 30 days with the actual environment $\left(R T T_{A C T}\right)$ compared to the optimized environment on the HPC $\left(R T T_{H P C}\right)$. As we can see, the time has been reduced significantly. Note that, even with these optimizations, the total computation time of our experiments is equivalent to 8 years, 337 days, and 12 hours on a single CPU core.

Even though an optimized test environment is required from an experimental standpoint, in practice, testing the firewall is just a single test activity in an array of test activities (e.g., testing the WAF, services, front-end). Given time and resource constraints, creating and maintaining test environments that are specific for each single test activity is very costly. Based on our experience, we found that test engineers prefer to test copies of the actual WAF configuration and services.

Since we, by design, optimized our test environment to enable large scale experiments, the test execution times in our experimental setting is not representative of test execution times in the actual environment (see Table 2). This is a problem as it biases the results of our experiments to the advantage of approaches that are less expensive in terms of test case generation but lead to the execution of more test cases, such as random testing. Therefore, in our analyses, we transform the time scale of the optimized environment into a realistic one, accounting for the actual test execution times in practice.

Assume $T=\left\{t_{1}, \ldots, t_{n}\right\}$ is a set of timestamps measured on the experimental environment, such that one timestamp is noted after the execution of every test. Then, for the $i$-th test case execution, $f\left(t_{i}\right)=t_{i}+\left(R T T_{A C T}-\right.$ $\left.R T T_{H P C}\right) * i$ transforms a timestamp $t_{i} \in T$ into the corresponding timestamp in the actual environment $\left(f\left(t_{i}\right)\right)$. 
TABLE 2

Average response time in milliseconds of some web service operations in our experimental environment compared to the case study's environment.

\begin{tabular}{l|r|r|r|r} 
& Op. 1 & Op. 2 & Op. 3 & Op. 4 \\
\hline$R T T_{H P C}$ & 11,36 & 11,39 & 11,46 & 16,61 \\
$R T T_{A C T}$ & 456,56 & 180,02 & 302,09 & 854,63
\end{tabular}

We will use the latter time scale to compare test strategies in a realistic fashion.

\subsection{Research Questions}

This work investigates several variants of a machine learning-driven testing strategy and a random testing strategy. We compare and evaluate all these strategies for their capability of finding bypassing attacks on both subject applications, i.e. ModSecurity and the proprietary WAF.

Since all testing strategies generate attacks from the same input space, i.e. the grammar introduced in section 3.1. we evaluate how efficient the different strategies are in sampling the input space for bypassing attacks. Therefore, we measure for each strategy how many distinct bypassing attacks are found over time.

RQ1: How efficient are ML-Driven E, ML-Driven $B, M L-D r i v e n$, and RAN in finding bypassing tests?

To assess the impact of the machine learning algorithms on the test result, we implemented two alternative classifiers for each of the ML-Driven strategies: RandomTree and RandomForest. As detailed in Section 3.3.3. both algorithms have complementary advantages and drawbacks. In brief, RandomForest is an ensemble classifier and, hence, is expected to be more robust against changes in the training set than RandomTree. However, RandomForest comes at a higher computational cost than RandomTree, since multiple models have to be learned. To assess the influence of both algorithms on our approach, we run ML-Driven with both algorithms and compare the number of identified bypassing attacks and path conditions in a given time budget.

RQ2: Does the choice of machine learning algorithm matter?

We evaluate whether, in our context, the benefits of the RandomForest compared to the RandomTree justify the increased computation overhead to learn the classifier. Therefore, we compare how many bypassing tests are found over time with these two algorithms. In addition, we assess whether the algorithms have an impact on the stability of the test result, i.e., we compare the variation among repetitions of the same test run.

RQ3: How does ML-Driven compare to similar techniques?

RQ3 compares our proposed technique with existing techniques for testing WAFs. We perform a quantitative comparison of the techniques by comparing the number of bypassing attacks found by each technique.

RQ4: Are we learning new, useful attack patterns as the number of distinct, bypassing attacks increases?

RQ4 assesses whether, as we find more bypassing tests, we also identify more attack patterns that can be useful to improve the rule set of the WAF. In our context, an attack pattern is the underlying root cause that enables an attack to bypass the WAF. For example, the attacks union $* ! 50000 *$ select pwd from user and union $* ! 50000 *$ select 99 share the pattern union $\star ! 50000 *$ select (root cause) while the remainder of the attacks differ. We want to investigate whether identifying successful attack patterns help understanding why attacks are bypassing and eventually fix the WAF to correctly detect further attacks. For the ML-Driven techniques, such a pattern is characterized by a path condition (see Def. 3). A path condition characterizes the slices, or combination of slices, that are likely causing an attack to bypass. Thus, a path condition represents a pattern that is not correctly detected by the WAF.

To answer RQ4, we measure how many path conditions can be extracted from a model that is learned by the ML-Driven techniques. More specifically, we analyze the growth in the number of path conditions as the number of successful, distinct attacks grows over time.

\subsection{Procedure}

We implemented the techniques proposed in this work into our SQLi testing tool called Xavier. Xavier supports the automated testing of web services for SQLi vulnerabilities and has been described in [10]. ML-Driven generates test cases in the form of SQLi attack strings, such as ' $\triangle O R " 1 "=" 1 " \#$. To generate malicious requests, Xavier takes such attack strings and injects them into sample SOAP messages, which are subsequently sent to an application under test. Xavier therefore relies on sample SOAP messages as inputs. They can be taken from existing web service test suites, or can easily be generated from the $\mathrm{WSDL}^{8}$ that describes the service interface under test.

In our experiments, when available, we use SOAP messages from the functional test suite of the service under test (Cyclos and our industrial case study) or, otherwise, we manually create SOAP messages from the WSDLs (HotelRS, SugarCRM). Each of these messages consists of a number of parameters and their legitimate values, which the services expect and that the WAF has to let through. In our testing process, each SOAP message is considered separately. A test generation technique, ML-Driven or RAN, continuously generates attacks, injecting one attack each time into a parameter of the selected SOAP message to create a new SOAP message, and then sends it to the web server.

Incoming SOAP requests to the web server are first treated by the WAF and only those that comply with firewall rules are forwarded to web applications, and otherwise are blocked. In case a request is blocked, the WAF replies to

8. http://www.w3.org/TR/wsdl 
the client that issued the request with a special response, stating that the request has been denied. When our testing tool, Xavier, receives such a response, it marks the test, embedded in the original request, with a blocked label " $\mathrm{B}$ " (for blocked), and otherwise a passed label " $\mathrm{P}$ " (bypassing).

\subsection{Parameter Setting}

For the ML-Driven approaches, there are various parameters to set for both the machine learning algorithms and $(\mu+\lambda)$-EA. For most of the parameters (e.g., machine learning training setting), we follow the recommendations from the literature [15], [28], [41]. Moreover, we used a trial-anderror procedure to calibrate the parameters $\mu$ and $\lambda$. The final parameter values are as follows:

Population size $(\mu)$. Typically, the population size for EAs can vary from tens to several thousands of individuals [41]. The best setting for this parameter depends on the nature of the problem and on the size of the search space [31]. In our case, the population size determines the number of tests that will be preserved for the next generation. A small population size may lead to loss of test cases with large bypassing probability, thus reducing the chances of generating new attacks able to pass through the WAF. From our preliminary experiments, we observed that a population size of 500 individuals works best for ML-Driven since it helps preserving as many as possible probable bypassing attacks.

Number of offsprings $(\lambda)$. In ML-Driven, newly generated offsprings (test cases) are used to update the archive, which is later used to retrain the classifier $D T$ by applying either RandomTree or RandomForest. Therefore, offsprings are the additional "data points" that are used for updating $D T$. A large $\lambda$ value means that in each generation a significant portion of the training set consists of new test cases; a small $\lambda$ value leads to retraining $D T$ with almost the same training set (i.e., updating $D T$ would be less effective). In this paper, we set $\lambda=4000$ to guarantee that $D T$ is always retrained with a large portion new test cases. While the total number offsprings is fixed, the number of offsprings generated from each test $t$ differs for the three variants of ML-Driven. For ML-Driven B and ML-Driven D, it is fixed and it is equal to $\operatorname{MAX}_{M}=10$ and $\operatorname{MAX}_{M}=100$, respectively. In $\mathrm{ML}-\mathrm{Driven} \mathrm{E}$, the number of offsprings for each test is determined dynamically using the adaptive strategy described in Section 3.4

Archive size. The archive keeps track of all attacks generated across the generations and it is used at the end of each generation as training set for machine learning. To avoid exceeding the resources of a typical laptop and to finish in a reasonable time we limit the training set to 6000 blocked attacks and 6000 bypassing attacks. If the number of tests in the archive is larger, we consider only the most recent tests, i.e., those produced in latest generations.

Stopping condition. The search terminates when the maximum search budget of 175 minutes is reached for ModSecure. For the proprietary WAF, we used a larger search budget of 500 minutes. To allow a fair comparison, RAN was configured with the same stopping condition.

Machine learning setting. For the machine learning algorithms used in this paper, i.e., RandomTree and Random-
Forest, we used their implementations available in Weka |28] with the default parameter values.

Number of repetition. To account for the randomization involved in the testing techniques (e.g., RAN), each algorithm was run 10 times for each subject application.

\subsection{Variables}

The following dependent variables are controlled or measured in our experiments:

- $\boldsymbol{D}_{\boldsymbol{t}}$ : The number of distinct tests that can bypass a target WAF at time $t$ is a way to measure the efficiency of a test strategy. Note that, given two distinct tests in the same attack category, one might be caught by the WAF while the other bypasses it. This may be caused by tests in a category that should be handled by different rules, some of them missing or incorrect in the current WAF rule set, or by an identical rule that is not general enough to block all tests in a category. Therefore, identifying similar but distinct bypassing tests is useful to identify attack patterns.

- $\quad D_{p c}$ : The number of distinct path conditions that can be extracted from decision trees. Each path condition characterizes a string pattern that a group of bypassing attacks has in common. Such string patterns can be added to the WAF rules to prevent further attack attempts containing the same pattern. Hence, $D_{p c}$ is a measure for how many attack patterns have been uncovered.

For each subject application, we ran each testing strategy 10 times and collected the number of distinct bypassing tests $\left(\boldsymbol{D}_{\boldsymbol{t}}\right)$ and distinct path conditions $\left(\boldsymbol{D}_{p c}\right)$ in each run. To this aim, every time a new test $t$ was generated, we collected the passing wall-clock time since the beginning of the search and then executed $t$ to see whether or not it could bypass the WAF. Therefore, we can compare the values of $\boldsymbol{D}_{\boldsymbol{t}}$ and $D_{p c}$ collected over time.

To verify whether $\boldsymbol{D}_{\boldsymbol{t}}$ scores significantly differ when using two different testing strategies (e.g., RAN and ML-Driven E), we used the Wilcoxon test with a significance level of $\alpha=0.05$. The Wilcoxon test is a non-parametric test and, thus, does not require the data to be normally distributed. For the statistically analysis, we collected the $D_{t}$ scores achieved by each alternative testing approach after intervals of 35 minutes for each repetition, resulting in five time windows for each run.

\section{Results}

In this section, we describe the results obtained in our case studies regarding the research questions formulated in Section 4.2 .

\subsection{RQ1: How efficient are $M L-D r i v e n ~ E$, $M L-D$ riven $B, M L-D$ riven $D$, and RAN in finding bypassing tests?}

To answer $R Q 1$, we applied the testing techniques to both subject applications and measured how many bypassing tests were found over time $\left(D_{t}\right)$. In particular, we compared 


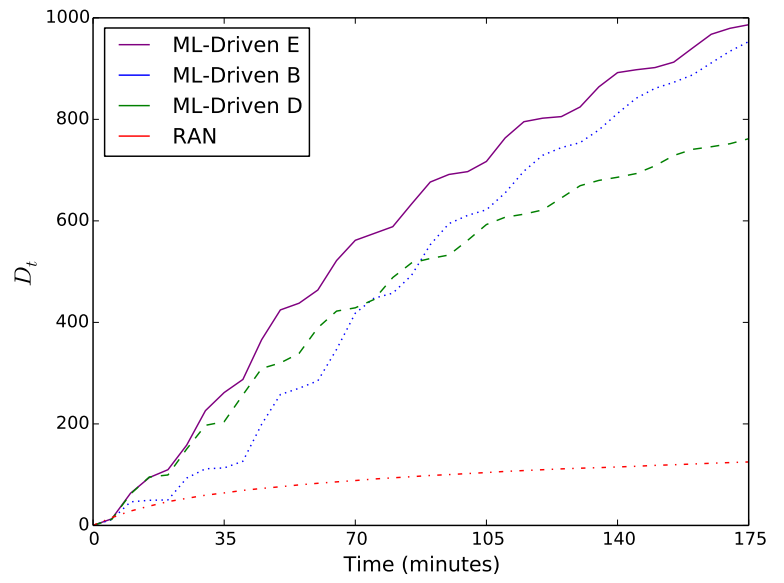

Fig. 5. Average number of bypassing tests $\left(D_{t}\right)$ found for nine tested operations (10 repetitions each) for ModSecurity.

the performance of the techniques based on the cumulative number of distinct bypassing tests generated over time. In the following, we discuss the results for ModSecurity and the proprietary WAF separately.

\subsubsection{Results for ModSecurity}

For the open-source WAF, we randomly selected nine parameters in total for testing, three parameters from each web application (HotelRS, SugarCRM, and Cyclos). For each technique, Fig. 5 depicts the average number of distinct, bypassing tests generated over time for ModSecurity measured within intervals of five minutes. The testing results for each individual parameter are in a technical report [8]. Fig. 6 depicts the same data as boxplots to help visualize statistical variation across the 10 repetitions. Table 3 reports the results of the Wilcoxon test by listing, in each cell, the test strategies that are significantly outperformed by the strategy matching the corresponding column.

The first observation is that all techniques can generate tests that bypass the WAF, suggesting that the WAF does not provide complete protection from SQLi attacks, putting online systems under its protection at risk. Further, by observing executed SQL statements on the database, we found that these bypassing tests can exploit SQLi vulnerabilities in HotelRS and SugarCRM. Second, the sharply increasing plots corresponding to ML-Driven E, ML-Driven B and ML-Driven $D$ indicate that they are much more efficient than RAN, the baseline for comparison. Overall, the results show that the ML-Driven techniques outperform RAN by an order of magnitude with respect to the number of distinct bypassing tests generated. The Wilcoxon test revealed that these differences were always statistically significant as depicted in Table 3 ( $p$-values $<0.01)$.

Among the machine learning-driven techniques, ML-Driven E constantly finds the most bypassing tests compared to ML-Driven B and ML-Driven D, which suggests that the adaptive budget allocation works well. The differences between ML-Driven $\mathrm{E}$ and its predecessors are always statistically significant in all the five time windows. One issue with ML-Driven D/B is that at the beginning of the test run, ML-Driven $\mathrm{D}$ finds

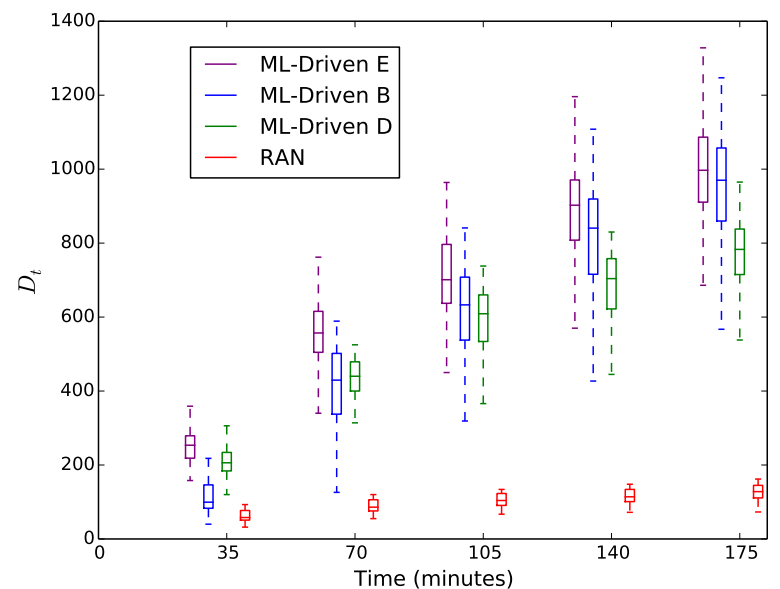

Fig. 6. Statistical variation for the number of bypassing tests found $\left(D_{t}\right)$ across all tested parameters for ModSecurity.

TABLE 3

Results of the Wilcoxon test for ModSecure. For each testing strategy (e.g., ML-E) we report whether it statistically outperforms the its counterparts (e.g., RAN) when $p$-values $<0.01$.

\begin{tabular}{c|c|c|c|c} 
Time Window & RAN $(\mathrm{p}<0.01)$ & ML-B $(\mathrm{p}<0.01)$ & ML-D $(\mathrm{p}<0.01)$ & ML-E $(\mathrm{p}<0.01)$ \\
\hline $35 \mathrm{~min}$ & & RAND & RAND & RAND \\
& & & ML-B & ML-B \\
& & & & RL-D \\
\hline $70 \mathrm{~min}$ & & RAND & RAND & ML-B \\
& & & & ML-D \\
\hline $105 \mathrm{~min}$ & & RAND & RAND & RAND \\
& & & & ML-B \\
& & & & ML-D \\
\hline $140 \mathrm{~min}$ & & RAND & RAND & RAND \\
& & ML-D & & ML-B \\
& & & & ML-D \\
\hline $175 \mathrm{~min}$ & & RAND & RAND & RAND \\
& & ML-D & & ML-B \\
& & & & ML-D
\end{tabular}

more bypassing tests, while this is the opposite later in the test run. More specifically, after a time window of 35 minutes ML-Driven $\mathrm{D}$ is statistically superior to ML-Driven B while between 70 and 105 minutes the two ML-Driven approaches are statistically equivalent; finally, in the last two time windows (i.e., 140 and 175 minutes), ML-Driven B statistically outperforms ML-Driven D. Since both techniques implement the same algorithm, this phenomenon can be attributed to a difference in the choice of parameters, or, more precisely, the parameter that determines the number of mutants generated per test (a detailed analysis is provided in a technical report [8]). While ML-Driven D and B generate a fixed number of mutants per test, ML-Driven $E$ adjusts the number of mutants in proportion to the test's bypassing probability. As a result, ML-Driven E spends the test budget more efficiently and finds bypassing attacks faster.

The plots for the ML-Driven techniques are also slightly oscillating, thus depicting the effect of iterative re-training of the classifier. The flat segments match the time intervals where the classifier is recomputed and no new tests are generated. The slopes of the plots tend to decrease over time as it becomes increasingly harder to find new bypassing tests that have not yet been executed. 


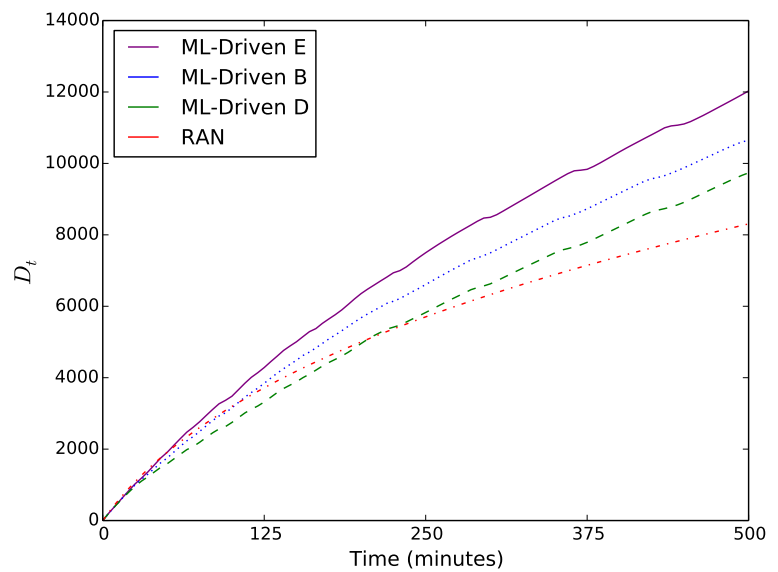

Fig. 7. Average number of bypassing attacks, proprietary WAF, all tested parameters.

\subsubsection{Results for the proprietary WAF}

We now address $R Q 1$ for the second subject application, the proprietary WAF. As for ModSecurity, all techniques are evaluated measuring the number of bypassing tests that are found over time.

Due to the considerably higher complexity of the WAF in the industrial case study, we selected a higher number of parameters for testing. Given that all testing strategies have to be applied to each parameter and each test run was repeated 10 times, testing all parameters would have been infeasible. Therefore, we selected one parameter for each distinct data type in the WSDL of the services under test, which resulted in a total of 75 parameters. The WAF in this case study determines the input validation routine to be executed for a parameter based mainly on the corresponding data type; hence selecting one parameter per data type maximizes the coverage of input validation routines.

Out of the 75 tested parameters, bypassing tests could be generated for 29 parameters. Each testing technique was able to generate bypassing tests for all of these 29 parameters. Fig. 7 depicts the average number of distinct bypassing tests per test strategy. The average is computed from all tested parameters and 10 repetitions per parameter.

Out of all techniques, ML-Driven E generates the most bypassing tests on average, followed by ML-Driven B and ML-Driven D. RAN finds the least bypassing tests. Since the statistical variation in the plots is high (see boxplot in Fig. 88, we separate the parameters into groups to better analyze the results. Parameters in the same group share similar input constraints in terms of number of allowed characters and tend to produce a similar number of bypassing test cases. It is worth noticing that this particular WAF considers not only such input constraints but also other criteria (e.g., SQLi blacklist). Therefore, parameters in a same group do not necessarily produce the same results. Table 4 shows the groups: Group 1 has two parameters that share an input constraint that restricts the number of characters to a maximum of eight. Similarly, Groups 2, 3, and 4 have similar constraints with 16,25 , and 35 characters, respectively. For each group, Table 5 reports the results of the Wilcoxon test when comparing the number of bypassing attacks generated

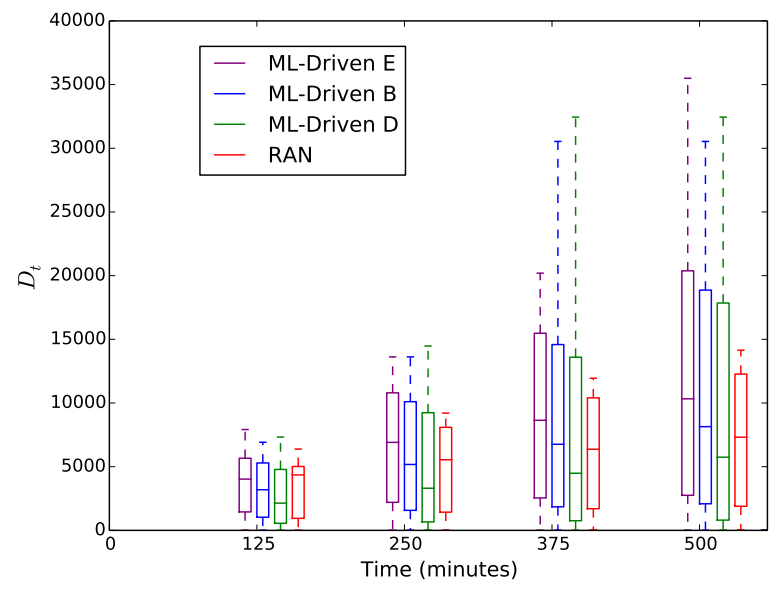

Fig. 8. Boxplots of the number of bypassing tests, all tested parameters, proprietary WAF.

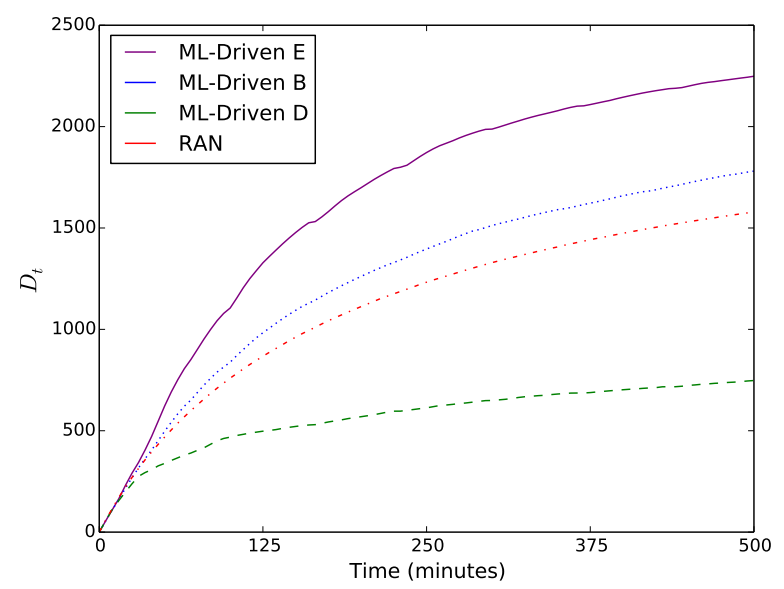

Fig. 9. Group 2: Average number of bypassing attacks, proprietary WAF, 7 parameters.

by the different testing strategies.

According to Table 5, we observe that for Groups 2, 3, and $4 \mathrm{ML}$-Driven $\mathrm{E}$ is always significantly superior to its predecessor as well as to RAN. However, looking at Fig. 9. 11 we notice the following trend: the more bypassing tests, the smaller the difference in test results across ML-Driven techniques. For example, ML-Driven E generates on average $+25 \%$ bypassing attacks compared to the second best approach in the comparison (i.e., ML-Driven B) for Group 2. For Group 3, the gap between ML-Driven E and the closest alternative approach (i.e., RAN) is $+30 \%$ on average. For Group 4, the average difference between ML-Driven E and its predecessor is lower than $10 \%$.

The average number of bypassing tests for the two

TABLE 4

Groups of parameters with a similar input constraint.

\begin{tabular}{l|r|l} 
& \#Parameter & Input Constraint \\
\hline Group 1 & 2 & Up to 8 Char. \\
Group 2 & 7 & Up to 16 Char. \\
Group 3 & 8 & Up to 25 Char. \\
Group 4 & 12 & Up to 35 Char.
\end{tabular}




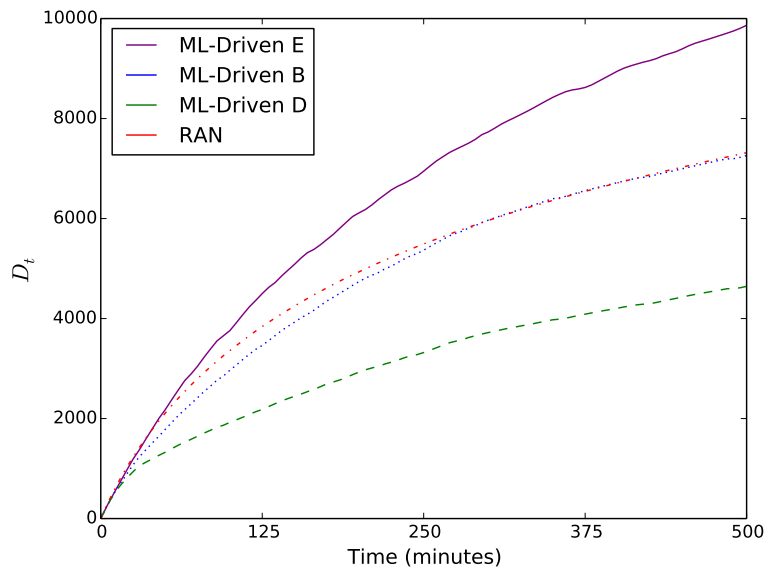

Fig. 10. Group 3: Average number of bypassing attacks, proprietary WAF, 8 parameters.

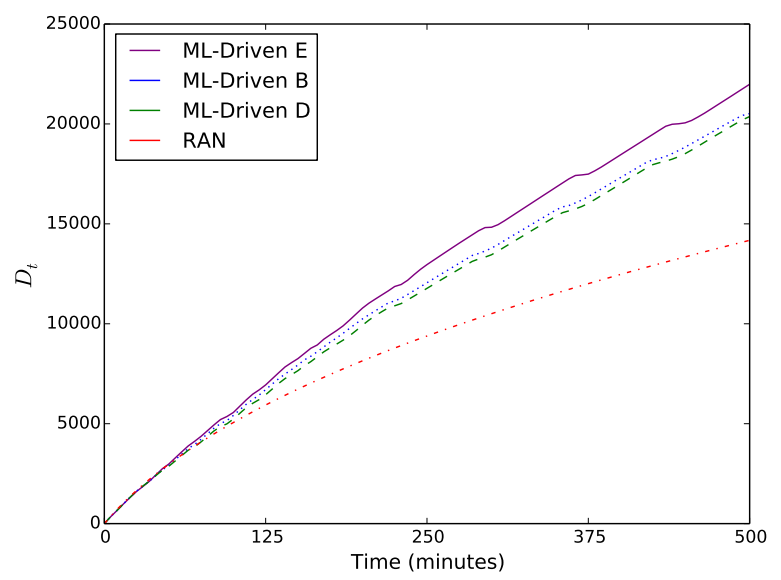

Fig. 11. Group 4: Average number of bypassing attacks, proprietary WAF, 12 parameters.

parameters in Group 1 is depicted in Fig. 12 All techniques tend to saturate after about 125 minutes and, after that, the number of bypassing tests increases very slightly. RAN is the most effective approach, significantly outperforming all ML-Driven variants after 105 minutes of running time (see Table 5). At the end of the search, RAN reaches up to approximately 40 bypassing tests while all the ML-Driven techniques find around 30 bypassing tests. The most prominent difference of this group, as compared to the others, is that very few bypassing tests are found. This is due to the fact that the number of allowed characters is only eight, thus resulting in a small number of attacks to bypass. Examining the training sets used to learn the classifiers more closely, we find that the training sets are heavily imbalanced. There are only between 15 and 30 bypassing attacks compared to up to 6000 blocked attacks, i.e., they represent only $0.25 \%-0.5 \%$ of the data points in the training set. For the other tested parameters, where the machine-learning driven techniques outperform RAN, there are typically several hundreds of bypassing attacks in a training set. As a result of the imbalanced training data, the learned classifier has a low recall $(\approx$ $80 \%$ ) and is less accurate in predicting an attack's bypassing

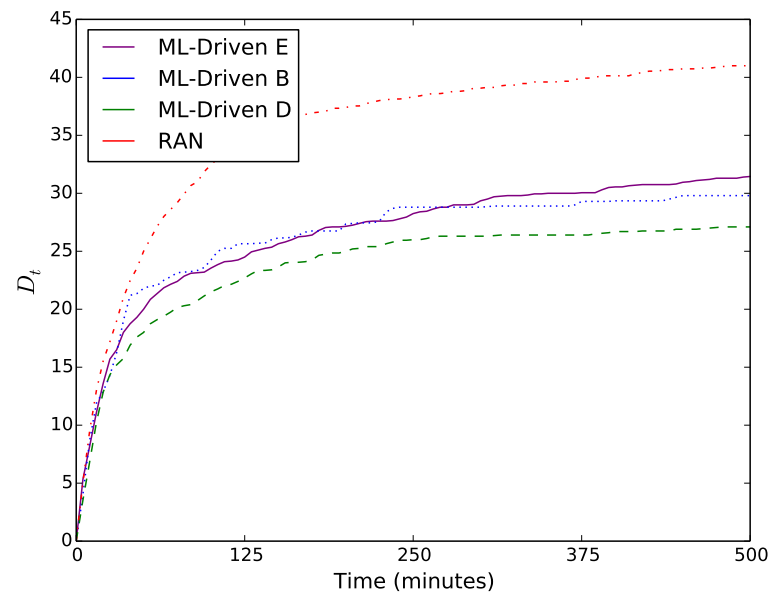

Fig. 12. Group 1: Average number of bypassing attacks, proprietary WAF, 2 parameters.

probability. RandomTree and RandomForest tend to generate a constant classifier in such a scenario, i.e., the resulting classifier likely labels all data tests as "blocked" because this leads to a very low classification error $(<1 \%)$.

Moreover, if we analyze the training sets used in consecutive retraining iterations (generations), we find that there are not substantially more bypassing attacks added to the training set. The quality of the classifier does not improve over the subsequent generations and is hardly worth the computation time necessary for retraining. To address this shortcoming, we conclude that the classifier should only be retrained if the training data has sufficiently improved. Otherwise, the existing classifier may be used to generate more offsprings until more bypassing attacks are found or the test budget is exceeded. In addition, in the presence of high imbalanced training sets, we may apply various well-known strategies in machine learning, such as data re-sampling strategies, weighted training, and penaltybased training. Investigating the best strategy to address the imbalance problem for ML-Driven is part of our future agenda.

\subsection{RQ2: Does the choice of machine learning algo- rithm matter?}

To answer RQ2, we run ML-Driven $\mathrm{E}$ with the chosen machine learning algorithms, namely RandomTree and RandomForest, and we compare the results. For this analysis, we focus on ML-Driven E only since it is the most efficient variant of ML-Driven according to the results discussed in Section 5.1 The evaluation is performed on the open-source case study (ModSecurity) in the same fashion as for RQ1.

Fig. 13 shows the average number of bypassing tests for nine selected parameters and 10 repetitions. The result indicates that ML-Driven $\mathrm{E}$ with RandomForest finds slightly more bypassing tests than ML-Driven E with RandomTree. However, the difference is not practically significant. The advantage of using a more stable classifier is partially lost due to the increased computation time for constructing this classifier. Therefore, although RandomForest may generate a more accurate/stable classifier than 
TABLE 5

Results of the Wilcoxon test for the proprietary WAF. For each testing strategy (e.g., ML-E) we report whether it statistically outperforms its counterparts (e.g., RAN) as well the corresponding $p$-values.

\begin{tabular}{|c|c|c|c|c|c|}
\hline Group & Time Window & RAN & ML-B & ML-D & ML-E \\
\hline & $35 \mathrm{~min}$ & & ML-D $(\mathrm{p}=0.01)$ & & \\
\hline \multirow{4}{*}{1} & $70 \mathrm{~min}$ & ML-D $(p=0.01)$ & ML-D $(p=0.03)$ & RAND $(\mathrm{p}<0.01)$ & \\
\hline & $105 \mathrm{~min}$ & $\begin{array}{l}\text { ML-B }(p=0.03) \\
\text { ML-D }(p=0.01) \\
\text { ML-E }(p<0.01)\end{array}$ & ML-D $(p=0.04)$ & & \\
\hline & $140 \mathrm{~min}$ & $\begin{array}{l}\text { ML-B }(\mathrm{p}<0.01) \\
\text { ML-D }(\mathrm{p}<0.01) \\
\text { ML-E }(\mathrm{p}<0.01)\end{array}$ & & & \\
\hline & $175 \mathrm{~min}$ & $\begin{array}{l}\text { ML-B }(\mathrm{p}<0.01) \\
\text { ML-D }(\mathrm{p}<0.01) \\
\text { ML-E }(\mathrm{p}<0.01)\end{array}$ & & & \\
\hline \multirow{5}{*}{2} & $35 \mathrm{~min}$ & ML-D $(\mathrm{p}<0.01)$ & RAND $(\mathrm{p}<0.01)$ & & $\begin{array}{l}\text { RAND }(\mathrm{p}<0.01) \\
\text { ML-B }(\mathrm{p}<0.01) \\
\text { ML-D }(\mathrm{p}<0.01)\end{array}$ \\
\hline & $70 \mathrm{~min}$ & ML-D $(p<0.01)$ & RAND $(\mathrm{p}<0.01)$ & & $\begin{array}{l}\text { RAND }(\mathrm{p}<0.01) \\
\text { ML-B }(\mathrm{p}<0.01) \\
\text { ML-D }(\mathrm{p}<0.01)\end{array}$ \\
\hline & $105 \mathrm{~min}$ & ML-D $(p<0.01)$ & RAND $(\mathrm{p}<0.01)$ & & $\begin{array}{l}\text { RAND }(\mathrm{p}<0.01) \\
\text { ML-B }(\mathrm{p}<0.01) \\
\text { ML-D }(\mathrm{p}<0.01)\end{array}$ \\
\hline & $140 \mathrm{~min}$ & ML-D $(p<0.01)$ & RAND $(\mathrm{p}<0.01)$ & & $\begin{array}{l}\text { RAND }(\mathrm{p}<0.01) \\
\text { ML-B }(\mathrm{p}<0.01) \\
\text { ML-D }(\mathrm{p}<0.01)\end{array}$ \\
\hline & $175 \mathrm{~min}$ & ML-D $(\mathrm{p}<0.01)$ & RAND $(\mathrm{p}<0.01)$ & & $\begin{array}{l}\text { RAND }(\mathrm{p}<0.01) \\
\text { ML-B }(\mathrm{p}<0.01) \\
\text { ML-D }(\mathrm{p}<0.01)\end{array}$ \\
\hline \multirow{5}{*}{3} & $35 \mathrm{~min}$ & $\begin{array}{l}\text { ML-B }(\mathrm{p}<0.01) \\
\text { ML-D }(\mathrm{p}<0.01)\end{array}$ & & & $\begin{array}{l}\text { RAND }(\mathrm{p}<0.01) \\
\text { ML-B }(\mathrm{p}<0.01) \\
\text { ML-D }(\mathrm{p}<0.01)\end{array}$ \\
\hline & $70 \mathrm{~min}$ & $\begin{array}{l}\text { ML-B }(\mathrm{p}<0.01) \\
\text { ML-D }(\mathrm{p}<0.01)\end{array}$ & & & $\begin{array}{l}\text { RAND }(\mathrm{p}<0.01) \\
\text { ML-B }(\mathrm{p}<0.01) \\
\text { ML-D }(\mathrm{p}<0.01)\end{array}$ \\
\hline & $105 \mathrm{~min}$ & $\begin{array}{l}\text { ML-B }(\mathrm{p}<0.01) \\
\text { ML-D }(\mathrm{p}<0.01)\end{array}$ & & & $\begin{array}{l}\text { RAND }(\mathrm{p}<0.01) \\
\text { ML-B }(\mathrm{p}<0.01) \\
\text { ML-D }(\mathrm{p}<0.01)\end{array}$ \\
\hline & $140 \mathrm{~min}$ & $\begin{array}{l}\text { ML-B }(\mathrm{p}<0.01) \\
\text { ML-D }(\mathrm{p}<0.01)\end{array}$ & & & $\begin{array}{l}\text { RAND }(\mathrm{p}<0.01) \\
\text { ML-B }(\mathrm{p}<0.01) \\
\text { ML-D }(\mathrm{p}<0.01)\end{array}$ \\
\hline & $175 \mathrm{~min}$ & $\begin{array}{l}\text { ML-B }(\mathrm{p}<0.01) \\
\text { ML-D }(\mathrm{p}<0.01)\end{array}$ & & & $\begin{array}{l}\text { RAND }(\mathrm{p}<0.01) \\
\text { ML-B }(\mathrm{p}<0.01) \\
\text { ML-D }(\mathrm{p}<0.01)\end{array}$ \\
\hline \multirow{5}{*}{4} & $35 \mathrm{~min}$ & & $\begin{array}{l}\text { RAND }(\mathrm{p}<0.01) \\
\text { ML-D }(\mathrm{p}<0.01)\end{array}$ & RAND $(\mathrm{p}<0.01)$ & $\begin{array}{l}\text { RAND }(\mathrm{p}<0.01) \\
\text { ML-B }(\mathrm{p}<0.01) \\
\text { ML-D }(\mathrm{p}<0.01)\end{array}$ \\
\hline & $70 \mathrm{~min}$ & & $\begin{array}{l}\text { RAND }(\mathrm{p}<0.01) \\
\text { ML-D }(\mathrm{p}<0.01)\end{array}$ & $\operatorname{RAND}(\mathrm{p}<0.01)$ & $\begin{array}{l}\text { RAND }(\mathrm{p}<0.01) \\
\text { ML-B }(\mathrm{p}<0.01) \\
\text { ML-D }(\mathrm{p}<0.01)\end{array}$ \\
\hline & $105 \mathrm{~min}$ & & $\begin{array}{l}\text { RAND }(\mathrm{p}<0.01) \\
\text { ML-D }(\mathrm{p}<0.01)\end{array}$ & RAND $(\mathrm{p}<0.01)$ & $\begin{array}{l}\text { RAND }(\mathrm{p}<0.01) \\
\text { ML-B }(\mathrm{p}<0.01) \\
\text { ML-D }(\mathrm{p}<0.01)\end{array}$ \\
\hline & $140 \mathrm{~min}$ & & $\begin{array}{l}\text { RAND }(\mathrm{p}<0.01) \\
\text { ML-D }(\mathrm{p}<0.01)\end{array}$ & RAND $(\mathrm{p}<0.01)$ & $\begin{array}{l}\text { RAND }(\mathrm{p}<0.01) \\
\text { ML-B }(\mathrm{p}<0.01) \\
\text { ML-D }(\mathrm{p}<0.01)\end{array}$ \\
\hline & $175 \mathrm{~min}$ & & $\begin{array}{l}\text { RAND }(\mathrm{p}<0.01) \\
\text { ML-D }(\mathrm{p}<0.01)\end{array}$ & RAND $(\mathrm{p}<0.01)$ & $\begin{array}{l}\text { RAND }(\mathrm{p}<0.01) \\
\text { ML-B }(\mathrm{p}<0.01) \\
\text { ML-D }(\mathrm{p}<0.01)\end{array}$ \\
\hline
\end{tabular}

RandomTree, its overhead negatively affects the number of generations (i.e., the number of tests to run against the WAF) that can be performed within the same amount of time (search budget). In other words, the gain in accuracy when using RandomForest does not compensate its overhead when used inside the test case generation loop. Even if less precise, RandomTree enables more iterations and an increased number of new test executions.

Fig. 14 shows a sample boxplot corresponding to the observed statistical variation for one representative parameter over 10 repetitions. We can see that the variation is small and similar for RandomForest and RandomTree. Similar results are observed for the other parameters. Therefore, to conclude, the choice between the two considered machine learning algorithms has no significant impact with respect to the number of bypassing tests or the degree of variation among repetitions.

\subsection{RQ3: How does ML-Driven compare to similar techniques?}

To answer RQ3, we compare our proposed technique ML-Driven with similar tools. The tools considered in this comparison are chosen according to the following selection criteria: Similar objective as ML-Driven, i.e., finding SQLi

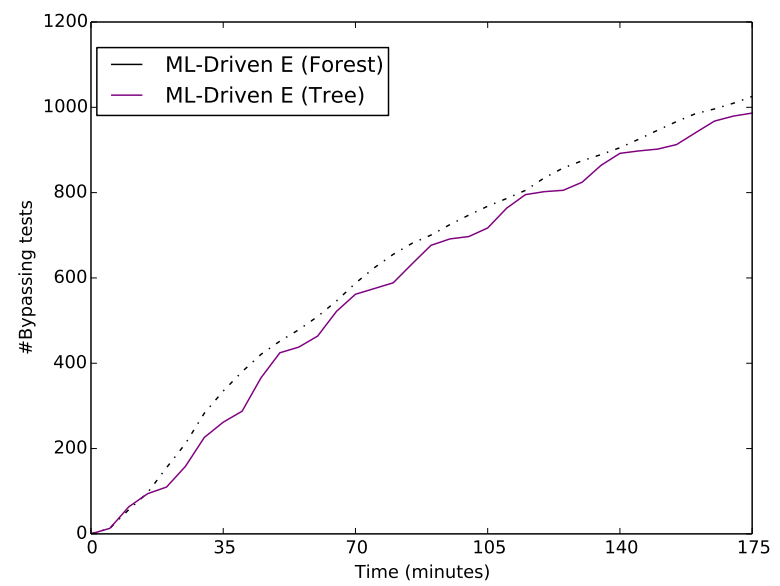

Fig. 13. Comparison of the RandomTree and RandomForest classifier. Average number of bypassing tests found for nine tested operations (10 repetitions each) in ModSecurity.

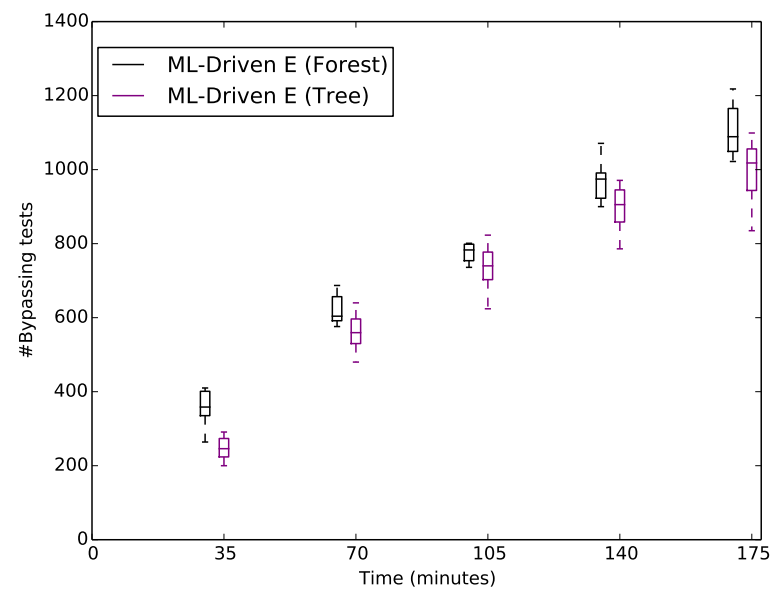

Fig. 14. Boxplots for the number of bypassing tests found with one representative parameter (get-relationships) in ModSecurity.

attacks that bypass a WAF under test; state-of-the-art covering a range of SQLi attacks and obfuscation methods; widely used or developed by known institutions or security experts. Following these criteria, we selected the WAF Testing Framework and SqlMap for comparison.

The WAF Testing Framework ${ }^{9}$ tests the attack detection capabilities of a WAF under test. It is a state-of-the-art tool developed by Imperva, which is the developer of a proprietary WAF and several other security solutions. The tool comes with a number of malicious HTTP requests that contain common SQLi attacks. To test a WAF, it submits the HTTP requests one by one to the WAF and checks if the malicious requests are blocked correctly.

SqlMap ${ }^{10}$ is a well-known penetration testing tool to detect SQLi vulnerabilities in web applications and services. It generates a large and diverse set of SQLi attacks, which cover all common SQLi attack techniques. To evade detection of WAFs, SqlMap uses several tamper scripts. Each

9. Downloaded from

https://www.imperva.com/Resources/FreeEvaluationTools

10. Version 1.0.7.42 downloaded from https://sqlmap.org 
TABLE 6

Tamper scripts of SqIMap selected for our experiments.

Tamper Script Name

between.py

commalesslimit.py

commalessmid.py

concat2concatws.py

equaltolike.py

greatest.py

halfversionedmorekeywords.py

ifnull2ifisnull.py

informationschemacomment.py

lowercase.py

modsecurityversioned.py

modsecurityzeroversioned.py

multiplespaces.py

randomcase.py

randomcomments.py

unionalltounion.py

uppercase.py

versionedmorekeywords.py
Short Description

Inserts a clause with the SQL keyword BETWEEN

Replaces instances like 'LIMIT M, N' with 'LIMIT N OFFSET M'

Replaces instances like 'MID(A, B, C)' with 'MID(A FROM B FOR C)'

Replaces instances like 'CONCAT(A, B)' with 'CONCAT_WS(MID(CHAR(0), 0, 0), A, B)'

Replaces all occurrences of operator equal (' $=$ ') with operator 'LIKE'

Replaces greater than operator ('>') with 'GREATEST' counterpart

Adds versioned MySQL comment before each keyword

Replaces instances like 'IFNULL(A, B)' with 'IF(ISNULL(A), B, A)'

Adds a comment to the end of all occurrences of "information_schema" identifier

Changes each keyword character to lower case

Surrounds the attack string with a MySQL version-specific comment (randomly generated version number)

Surrounds the attack string with a MySQL version-specific comment (version number set to 0)

Adds multiple spaces around SQL keywords

Changes randomly the case of letters in a SQL keyword

Add random comments to SQL keywords

Replaces UNION ALL SELECT with UNION SELECT

Changes each keyword character to upper case

Surrounds each keyword with a version-specific MySQL comment
TABLE 7

Results for testing the proprietary WAF with the WAF TestingFramework and SqIMap.

\begin{tabular}{l|r|r|r|r} 
& \multicolumn{2}{|c}{ WAF Testing Framework } & \multicolumn{2}{c}{ SqlMap } \\
Parameter & \multicolumn{1}{|c}{ \#T $C_{\text {Total }}$} & $\# T C_{\text {Bypass }}$ & $\# T C_{\text {Total }}$ & $\# T C_{\text {Bypass }}$ \\
\hline Group 1 & 19 & 0 & 3283.9 & 0.0 \\
Group 2 & 19 & 0 & 3281.6 & 0.0 \\
Group 3 & 19 & 0 & 3278.9 & 0.3 \\
Group 4 & 19 & 3 & 3260.5 & 66.5
\end{tabular}

tamper script applies a specific obfuscation method to a SQLi attack (e.g. randomly changes the case of letters in a SQL keyword). Since some tamper scripts are only meant to be applied with particular WAFs, web application frameworks (e.g. ASP or PHP), or database management systems, we selected a number of tamper scripts that are applicable to our subject applications (i.e., applicable to the proprietary WAF/ModSecurity, SOAP web services written in PHP/Java, and MySQL). Table 6 lists the tamper scripts selected for our experiments and gives a brief description for each. Note that SqlMap does not report how many or which attacks bypassed a WAF under test. Therefore, we routed the HTTP traffic between SqlMap and the WAF under test through a custom HTTP proxy, which determines whether each generated attack bypassed the WAF or was blocked.

To compare ML-Driven with the WAF Testing Framework and SqlMap, we use both tools to test ModSecurity and our industry partner's proprietary WAF. The configuration of both WAFs is the same as in the other reported experiments. Since SqlMap's attack generation is randomized, we repeated each experiment involving SqlMap 10 times and report the averages. The attack generation in the WAF Testing Framework is deterministic and, hence, we report the result of a single run. We do not impose any limit on the number of generated test cases; both tools are configured to execute as many test cases as they are capable of generating.

For the experiment with the proprietary WAF, we randomly selected from each of the four parameter groups (refer to Table 4) one representative parameter to test. We found in the evaluation of RQ1 that parameters in a same group share a similar number of bypassing attacks. Given how computation intensive these experiments are, we test only a representative parameter of each group and interpret the result as a performance indicator for the other parameters in the same group. Table 7 reports for both tools the total number of generated test cases $\left(T C_{\text {Total }}\right)$ and the number of test cases bypassing the proprietary WAF $\left(T C_{\text {Bypass }}\right)$. The WAF Testing Framework executes for each tested parameter 19 test cases. For the tested parameter of Group 4, three of the 19 attacks are bypassing. For all other tested parameters, the WAF Testing Framework does not find a bypassing attack. SqlMap generates a similar number of test cases for each tested parameter (on average between 3260.5 and 3283.9 test cases). For the parameter of Group 4, SqlMap finds on average 66.5 bypassing attacks and for the parameter of Group 3, SqlMap finds on average only 0.3 bypassing attacks. For the other parameters, SqlMap does not find any bypassing attacks.

The fact that both tools find the most bypassing attacks for Group 4 can be explained with the fact that the input length constraint for this group is the least strict (refer to Table 44. This also confirms the trend observed in the results for ML-Driven, which found the most bypassing attacks for Group 4 as well. For the other groups, the WAF Testing Framework and SqlMap do not reliably find bypassing attacks, because the generated attacks are either too long to satisfy the input length constraint or contain a pattern that is blacklisted.

For the experiment with ModSecurity, we use the WAF Testing Framework and SqlMap to test the same nine parameters as selected for the evaluation of ML-Driven (refer to RQ1). The WAF Testing Framework executes again 19 test cases per tested parameter and SqlMap executes a similar number of test cases as in the previous experiment $(\sim 3270$ test cases per parameter). However, neither the WAF Testing Framework nor SqlMap find any bypassing attack for any of the tested parameters.

When comparing the results of the WAF Testing Framework and SqlMap with ML-Driven (refer to Section 5.1), we find that the formers are either completely ineffective or significantly less effective than ML-Driven. For most of the tested parameters, both tools fail to find any bypassing 


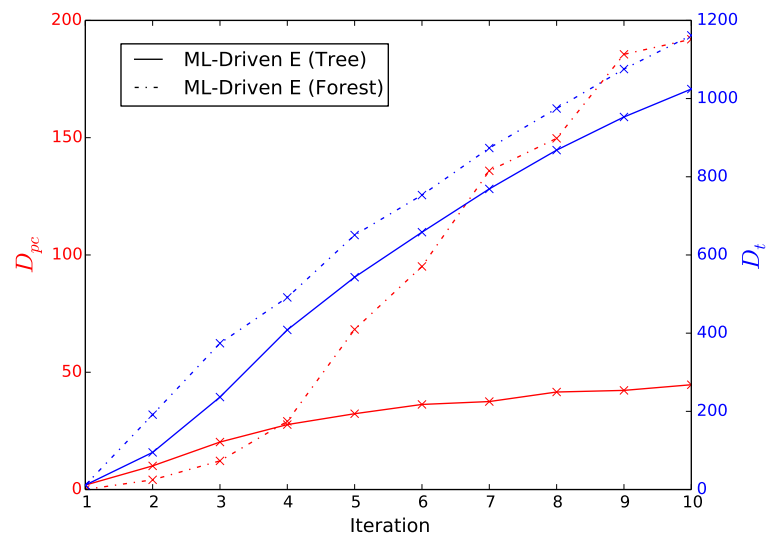

Fig. 15. Number of path conditions (red y-axis on the left) and bypassing tests (blue $y$-axis on the right) for ML-Driven $\mathrm{E}$ with RandomTree and RandomForest.

attack, while ML-Driven finds a considerable number of them. For the few parameters, for which the alternative tools find bypassing attacks, ML-Driven finds an order of magnitude more bypassing attacks. Furthermore, the attacks found by the alternative tools are a subset of the attacks found by ML-Driven and, hence, using the alternative tools in combination with ML-Driven would not provide more distinct bypassing attacks. Another advantage of ML-Driven over the alternative tools is that ML-Driven abstracts path conditions from the bypassing attacks, which helps the firewall administrator to repair the WAF (refer to Section 6.

\subsection{RQ4: Are we learning new, useful attack patterns as the number of distinct, bypassing attacks increases?}

As explained in Section 3.3 , the ML-Driven techniques periodically build a model (i.e., decision trees) that is used to guide the generation of new attacks. However, this model can also be used to abstract common string patterns shared by and possibly causing bypassing attacks. Therefore, for RQ4 we analyze the models that are learned during the test runs of ML-Driven E. For both RandomForest and RandomTree, we analyze how the number of path conditions increases as the result of generating more distinct bypassing attacks.

Fig. 15 depicts the average number of path conditions that can be extracted from a model (red) and the number of bypassing tests with which the model is trained (blue). Since the ML-Driven techniques retrain the model at the end of each generation, the corresponding training iterations (or EA generations) are depicted on the horizontal axis. These curves are based on the average of all test runs performed with the ModSecurity case study.

The number of distinct, bypassing tests, which are used to train the model, is steadily increasing for both techniques. This is due to fact that the further a test run progresses, the more bypassing tests are found and are used, in turn, to retrain the model. The number of path conditions that can be extracted from a model is also steadily increasing for both techniques, although beginning from generation 4 , there are significantly more path conditions extracted with
TABLE 8

Slice encodings.

\begin{tabular}{l|r|l|l|r} 
Slice & Literal & & Slice & Literal \\
\hline$S 1 d$ & or & & $S b$ & $!$ \\
$S f 1$ & or 1 & & $S 3$ & 1 \\
$S 5$ & $/^{* *} /$ & & $S 6$ & $\sim 1$ \\
$S 26$ & $\#$ & & $S 57$ & $\sqcup 1$ \\
$S 2$ & $\sqcup$ & $S 29$ & $\sqcup$ \\
$S 0$ & 0 & & $S 15$ & ) \\
$S f$ & true & & $S c$ & $"$
\end{tabular}

RandomForest than with RandomTree. For RandomForest, the number of path conditions is close to 200 after 10 generations, compared with 50 for RandomTree.

For both techniques, the number of bypassing tests used to train the model and the number of path conditions obtained from the model are concurrently increasing. This is no surprise as more test cases lead to refined models. For RandomForest, that leads to even more path conditions since many more trees are built.

In general, path conditions can help determine the reason why a set of attacks is bypassing. This knowledge can be utilized to stop further attack attempts from succeeding by inferring a string pattern from the path condition that matches all the attacks described by it. Such a string pattern can be in turn added to the WAF's rule set to block all further attacks containing the same string pattern.

To better explain the benefits of having more attack patterns, let us describe examples of path conditions and the attacks they characterize that are obtained from a test run of the industrial WAF used in our evaluation. In particular, let us consider an example of a path condition: $p c_{1}=$ $S 1 d \wedge S f 1 \wedge S 5 \wedge \neg S 11 \wedge \neg S 25 \wedge \neg S 26 \wedge \neg S 1 b \wedge \neg S 15 \wedge \neg S 2 f$. Table 8 shows the literals that are represented by the corresponding slices. An example attack characterized by $p c_{1}$ is 0 or $1 / * * /$, which is an obfuscated variation of a tautology attack, e.g. " or $1=1$. All attacks characterized by $p c_{1}$ contain the slices $S 1 d, S f 1$ and $S 5$. If a WAF blocks inputs containing any of the three slices, the attacks characterized by $p c_{1}$ do no longer bypass.

As shown by our quantitative analysis, the number of discovered path conditions increases during test execution. With more path conditions, the reasons why attacks are bypassing can be more precisely characterized. To illustrate this phenomenon, we analyze the path conditions obtained in different generations of the same test run. To limit the number of path conditions in this example, we only consider path conditions that contain the slice $S 1 d$, like in $p c_{1}$. This slice represents the SQL keyword or and can for example be part of a SQL tautology attack, e.g., " or $1=1$.

The previously analyzed path condition $p c_{1}$ is obtained from the first generation of a test run. In contrast, Table 10 shows more path conditions from the same test run obtained after the fifth generations. Table 9 depicts the string patterns that can be devised from the path conditions.

The first notable difference between both sets of path conditions is their number. In the first generation, only one path condition contains slice $S 1 d$ while there are eight of them by the fifth generation. When analyzing the patterns identified by the path conditions, there are several interesting observations. First, $p c_{2}$ to $p c_{7}$ identify patterns of 
TABLE 9

This table shows the pattern learned from each path condition and example attacks containing the pattern (the pattern characterized by the path conditions is highlighted in red).

\begin{tabular}{|c|c|c|c|}
\hline Generations & Id & Pattern & Example SQLi Attack \\
\hline 1 & $p c_{1}$ & or $1, /^{* *} /$ & 0 or $_{1} 1 / * * /$ \\
\hline 5 & $p c_{2}$ & or, 1 & 'or true like 11||$^{\prime}$ \\
\hline 5 & $p c_{3}$ & or, ), \# & ') or not false\# \\
\hline 5 & $p c_{4}$ & or, 0 & 1 or true $>(0)$ \\
\hline 5 & $p c_{5}$ & or, true, \# & 'or w true\# \\
\hline 5 & $p c_{6}$ & or, 0 & 0) or ! "-- \\
\hline 5 & $p c_{7}$ & or, " & " I | true or" \\
\hline 5 & $p c 8$ & or, $\left.\right|^{* * *} /, \#, u^{\prime} 0, !$ & or $/ * * / ! 0$ is true\# \\
\hline 5 & $p c_{9}$ & or, $/ * * /, 1, \sim 1$ & 1 or $/ * * / 0<\left(\sim \_1\right)$ \\
\hline
\end{tabular}

bypassing attacks that use slice $S 1 d$, but require $S 5$ to be absent $(\neg S 5)$, thus, suggesting the pattern identified in the first generation was incomplete. For example, $p c_{5}$ characterizes bypassing attacks containing slice $S 1 d$ combined with the boolean identifier true and the comment symbol \#, e.g., " or true\#. Such attacks are not matched by the pattern represented by $p c_{1}$ and, thus, would still bypass the WAF if only $p c_{1}$ would be considered in fixing the WAF.

To conclude, we see from the example above that having more path conditions helps derive a better understanding of the patterns shared by bypassing attacks. In turn, this puts a firewall administrator in a better position to devise an effective patch for a WAF's rule set, as we will demonstrate in Section 6

\section{USING ATTACK PATTERNS TO REPAIR A WAF}

In this section, we demonstrate how the identified bypassing attacks and corresponding path conditions can be used to improve the WAF's rule set. We also discuss the benefits of ML-Driven E compared to the other ML-Driven strategies, RAN, SqlMap and WAF Testing Framework.

\subsection{Repair Strategy}

Typically, a rule set uses regular expressions to match known malicious attacks (refer to Section 2.1. Our goal is to modify these regular expressions in such a way that the bypassing attacks found by ML-Driven do no longer bypass the WAF. We investigated a first attempt to semi-automate the WAF repairing process in our recent work [11]. In particular, we use multi-objective optimization algorithms to optimize two goals: (i) maximizing the number of blocked attacks and (ii) minimizing the number legitimate requests being blocked (false positives).

In this paper, we consider a general strategy for modifying the regular expressions as outlined in Algorithm 5 While parts of the strategy can be automated [11], regular expressions derived from attack patterns (line 8 and 9 still require manual inspection and adaptation depending on the WAF under evaluation. The required inputs for the strategy are the test outputs of ML-Driven, i.e., a set $A$ of bypassing attacks and a set $P C$ of path conditions learned from $A$, and a set $R$ of regular expressions used by the WAF under test to match known attacks. Note that the attacks in $A$ are not matched by any regular expression in $R$, otherwise the attacks would not bypass in the first place, and, hence, $\forall a \in A \nexists r \in R: r$ matches $a$. The output of the strategy is a
Algorithm 5 A strategy to repair a faulty WAF rule set using bypassing attacks and path conditions.

1: Inputs:

$A$ : a set of bypassing attacks such that $|A|>0$

$P C$ : a set of path conditions learned from $A$

$R$ : a set of regular expressions such that

$\forall a \in A \nexists r \in R: r$ matches $a$

2: Output:

$R^{\prime}$ : a set of regular expressions such that

$\forall a \in A \exists r \in R^{\prime}: r$ matches $a$

3: $R^{\prime} \leftarrow R$

4: while $P C \neq \emptyset$ do

5: $\quad s \leftarrow$ selectMostFrequentSlice $(P C)$

6: $\quad P C_{s} \leftarrow$ getPathConditionsContainingSlice $(s)$

7: $\quad A_{s} \leftarrow$ getCharacterisedAttacks $\left(P C_{s}\right)$

8: $\quad$ Select $r_{i} \in R$ that matches attacks similar to $A_{s}$

9: $\quad$ Modify rule $r_{i}$ to $r_{i}^{*}$ such that it matches $A_{s}$

10: $\quad R^{\prime} \leftarrow\left(R^{\prime} \backslash\left\{r_{i}\right\}\right) \cup\left\{r_{i}^{*}\right\}$

11: $\quad P C \leftarrow P C \backslash P C_{s}$

12: end while

13: return $R^{\prime}$

set of regular expressions $R^{\prime}$, which results from modifying some regular expressions in $R$ such that they match all attacks in $A$. We will use a running example in which $A$ and $P C$ are initialized with the attacks and path conditions from generation five of the previous examples (refer to Table 9). $R$ is initialized with the regular expressions from the proprietary WAF used in our evaluation. Note that the examples are not artificial as the regular expressions were actively protecting the industrial web service application used in our evaluation. The attacks in Table 9 would have bypassed the WAF deployed in the production system.

The strategy starts in line 3 by initializing the output variable $R^{\prime}$ by assigning $R$ to it. The while loop from line 4 to 12 is repeated until all path conditions in $P C$ are processed. In line 5 , the loop begins by selecting a slice $s$ that is the most frequently contained slice among the path conditions in $P C$. Line 6 selects all path conditions $P C_{s} \subset P C$ that contain slice $s$. Then, line 7 selects all attacks $A_{s} \subset A$ that are characterized by the path conditions in $P C_{s}$. In the running example, the slice $S 1 d$ is assigned to $s$ because it is part of eight path conditions ( $p c_{2}$ to $\left.p c_{9}\right)$ and, hence, is part of more path conditions than any other slice. The path conditions $p c_{2}$ to $p c_{9}$ (see Table 10 ) are assigned to $P C_{s}$ because they contain slice $S 1 d$. Accordingly, the attacks characterized by the path conditions $p c_{2}$ to $p c_{9}$ are assigned to $A_{s}$ (refer to Table 9 .

In line 8 a security analyst manually inspects the regular expressions in $R$ and identifies a regular expression $r_{i}$ that should be modified in order to correctly identify the bypassing attacks in $A_{s}$. Depending on the regular expressions $R$, there might already exist an expression $r_{i} \in R$ that identifies attacks similar to those in $A_{s}$ and, hence, could be modified to also account for $A_{s}$. Alternatively, if no such regular expression exists, a new regular expression can be created or, if there is more than one candidate regular expression for modification, several regular expressions can be modified so that each expression matches a subset of $A_{s}$. The security analyst is free to make this decision based on how he intends to logically structure the rule set. In the running example, 
TABLE 10

Path Conditions from generation 5.

\begin{tabular}{l|l} 
Id & Path condition \\
\hline$p c_{2}$ & $S 1 d \wedge S 57 \wedge \neg S 25 \wedge \neg S 26 \wedge \neg S c \wedge \neg S 14 \wedge \neg S 1 \wedge \neg S 3 e \wedge \neg S 0 \wedge \neg S 38 \wedge \neg S 29 \wedge \neg S 5 \wedge \neg S e 1 \wedge \neg S 1 c \wedge \neg S 1 d e \wedge \neg S 76 \wedge \neg S 67$ \\
$p c_{3}$ & $S 1 d \wedge S 26 \wedge S 15 \wedge \neg S f \wedge \neg S 1 c \wedge \neg S e 1 \wedge \neg S 25 \wedge \neg S 5 \neg S c \wedge S 14 \wedge \neg S 1 d e \wedge \neg S 3 \wedge \neg S 76 \wedge \neg S 29 \wedge \neg S 38 \wedge \neg S 67$ \\
$p c_{4}$ & $S 1 d \wedge S 29 \wedge \neg S e 1 \wedge \neg S 25 \wedge \neg S 5 \wedge \neg S 14 \wedge \neg S 1 d e \wedge \neg 3 e \wedge \neg S 76 \wedge \neg S 38 \wedge \neg S 67$ \\
$p c_{5}$ & $S 1 d \wedge S f \wedge S 26 \wedge \neg S 1 c \wedge \neg S e 1 \wedge \neg S 25 \wedge \neg S 5 \wedge \neg S c \wedge \neg S 14 \wedge \neg S 1 d e \wedge \neg S 3 e \wedge \neg S 76 \wedge \neg S 29 \wedge \neg S 38 \wedge \neg S 67$ \\
$p c_{6}$ & $S 1 d \wedge S 0 \wedge \neg S 25 \wedge \neg S 26 \wedge \neg S c \wedge \neg S 14 \wedge \neg S 3 e \wedge \neg S 3 \wedge \neg S 29 \wedge \neg S 5 \wedge \neg S e 1 \wedge \neg S 1 c \wedge \neg S 1 d e \wedge \neg S 76 \wedge \neg S 67$ \\
$p c_{7}$ & $S 1 d \wedge S c \wedge \neg S 1 c \wedge \neg S 1 \wedge \neg S 25 \wedge \neg S 5 \wedge \neg S 14 \wedge \neg S 1 d e \wedge \neg S 3 e \wedge \neg S 76 \wedge \neg S 29 \wedge \neg S 38 \wedge \neg S 67$ \\
$p c 8$ & $S 1 d \wedge S 5 \wedge S 26 \wedge S 2 \wedge S 0 \wedge S b \wedge \neg S a 8 \wedge \neg S 7 c 3 \wedge \neg S f \wedge \neg S 1 e 1 \wedge \neg S 25 \wedge \neg S d \wedge \neg S c \wedge \neg S 14 \wedge \neg S 15 \wedge \neg S 26 d \wedge \neg S 60 \wedge \neg S 2 e \wedge$ \\
& $\neg S 2 d \wedge \neg S 38 \wedge \neg S 3$ \\
$p c_{9}$ & $S 1 d \wedge S 3 \wedge S 5 \wedge S 6 \wedge \neg S f \wedge \neg S 25 \wedge \neg S f 1 \wedge \neg S c \wedge \neg S 26 \wedge \neg S 1 b \wedge \neg S 5 a \wedge \neg S 14 \wedge \neg S 71$ \\
\hline
\end{tabular}

we assign the following regular expression to $r_{i}$ :

$$
\text { (? i ) ' }[\backslash s]+\text { or }
$$

The regular expression begins with (?i), which enables case-insensitive matching. It matches strings that contain an apostrophe followed by at least one whitespace $\left([\backslash s]^{+}\right)$ followed by the SQL keyword or. We select this expression for modification because it is the only expression in the rule set that tries to match attacks containing the SQL keyword or. Since all attacks in $A_{s}$ also contain or, the expression is a suitable candidate for modification.

In line 9 . the security analyst extends the regular expression $r_{i}$ so that it also matches the attacks in $A_{s}$. The path conditions in $P C_{s}$ provide guidance on how $r_{i}$ could be extended. In the running example, when we contrast the expression $r_{i}$ with the path conditions in $P C_{s}$, we make several observations regarding why $r_{i}$ does not identify the attacks in $A_{s}$ : (i) unlike in $r_{i}$, not all attacks in $A_{s}$ start with an apostrophe, but they start either with an apostrophe, a double quote (e.g. $p c_{7}$ ), or a digit (e.g. $p c_{6}$ ); (ii) unlike in $r_{i}$, none of the path conditions requires a leading whitespace before the literal or; and (iii) $r_{i}$ does not account for SQL comment characters, i.e., \# and $/ \star \star /$, which are frequently used in bypassing attacks (e.g. $p c_{3}, p c_{5}, p c_{8}$, and $p c_{9}$ ). Based on these observations, we devise an improved regular expression $r_{i}^{*}$ to avoid the mentioned shortcomings:

$$
\text { (?i) ( ( '|"|\d).*or)|(or.*(/**/|\#)) }
$$

The modified expression $r_{i}^{*}$ consists of two parts. The first part is $\left(('|"| \backslash d) .^{*}\right.$ or $)$ and addresses (i) as well as (ii). It addresses (i) by replacing the apostrophe with $('|"| \backslash d)$ and it addresses (ii) by replacing $[\backslash s]+$ with an arbitrary character sequence (denoted by .*). Therefore, the first part matches strings that contain either an apostrophe, a double quote, or a digit, followed by an arbitrary character sequence, and followed by or. The second part is (or.* $(/ * * / \mid \#))$ and addresses (iii) as it matches strings that contain or and a SQL comment character $(/ * * /$ or \#). Since both parts are joined with an alternation $(\mid)$, the strings matched by $r_{i}^{*}$ is the union set of the strings matched by the first part and the second part.

With the performed changes, $r_{i}^{*}$ matches all attacks in $A_{s}$. In line 11, we remove the old expression $r_{i}$ from the output set $\vec{R}^{\prime}$ and add $r_{i}^{*}$ to $R^{\prime}$. Since all attacks in $A_{s}$ are now correctly identified, we remove in line 11 the path conditions $P C_{s}$ from $P C$ and continue with the next loop iteration until all path conditions in $P C$ are processed. In the running example, $P C$ is empty after the first iteration and the main loop ends.

Note that the proposed strategy helps a security expert efficiently deal with the typically large number of path conditions found by ML-Driven by grouping conditions containing similar attack patterns. Grouping patterns helps the security experts to consider relevant patterns together when devising an improved regular expression and, hence, they do not have to consider each pattern individually. The strategy is defined in such a way that the most frequent attack patterns are tackled first.

\subsection{Suitability of other techniques for the purpose of repairing a WAF}

The testing techniques and tools discussed throughout this paper find bypassing attacks with varying effectiveness. This section discusses how suitable the different techniques are for the purpose of repairing a WAF.

In our experiments, SqlMap and the WAF Testing Framework did find none or very few bypassing attacks and, hence, do not provide much useful information for repairing a WAF as many bypassing attacks would remain uncovered. RAN is more effective in finding bypassing attacks than SqlMap and the WAF Testing Framework, but it does not identify common bypassing attack patterns. In absence of such patterns, a security analyst has to translate a possibly large number of bypassing attacks into an WAF patch. This limitation affects not only RAN but also the other tools (i.e., SqlMap and WAF Testing Framework) as they provide the set of bypassing attacks without any additional information about which substrings are associated with bypassing the WAF. Instead, our ML-Driven techniques provide both the set of bypassing attacks (which are larger in number) and the associated attack patterns identified by machine learning algorithms (i.e., RandomTree and RandomForest).

Amongst the ML-Driven techniques, ML-Driven $\mathrm{E}$ was found to be the most efficient. Having more successful bypassing attacks provides additional benefits for security analysts in charge of repairing the vulnerable WAF. First, a lower number of distinct attacks will lead to less attack patterns being identified and less new regular expressions to be added to the WAF rule set. Therefore, ML-Driven B/D may miss some distinct attacks, leading to less robust patches as the fixed WAF would not be able to detect/match the missed patterns. Second, machine learning algorithms better identify useful and useless attack patterns (or path conditions) when more bypassing attacks are provided as shown in Section 5.4. For example, let us assume that ML-Driven B/D generate one single bypassing attack with 
TABLE 11

Comparison of the attack patterns found by ML-Driven E, ML-Driven B and ML-Driven D.

\begin{tabular}{|c|c|c|c|c|}
\hline Id & Pattern & ML-E & ML-B & ML-D \\
\hline$p c_{1}$ & or $1,1^{* *}$ & $\sqrt{ }$ & $\checkmark$ & $\sqrt{ }$ \\
\hline$p c_{2}$ & or, 1 & $\checkmark$ & $\checkmark$ & $x$ \\
\hline$p c_{3}$ & $o r,)^{\prime}, \#$ & $\checkmark$ & $\checkmark$ & $\checkmark$ \\
\hline$p c_{4}$ & or, ${ }^{\circ} 0$ & $\checkmark$ & $\checkmark$ & $\checkmark$ \\
\hline$p c_{5}$ & or, true, \# & $\checkmark$ & $x$ & $x$ \\
\hline$p c_{6}$ & or, 0 & $\checkmark$ & $\checkmark$ & $\checkmark$ \\
\hline$p c_{7}$ & or, " & $\checkmark$ & $\checkmark$ & $\checkmark$ \\
\hline$p c_{8}$ & or, $\left.\right|^{* * *} /, \#, \cup^{\prime} 0, !$ & $\checkmark$ & $x$ & $x$ \\
\hline$p c_{9}$ & or $, /^{* * *} /, 1, \sim 1$ & $\checkmark$ & $\checkmark$ & $x$ \\
\hline
\end{tabular}

the following slices: $S_{1}=$ ", $S_{2}={ }^{\prime}, S_{3}=" a "=" a ", S_{4}=\#$. With one single attack, it may be difficult for security analysts to determine which of these slices is actually associated with bypassing the WAF. Therefore, deeper analysis (or more attacks) is needed to determine which slices should form regular expressions to add to the WAF's rule set.

To illustrate the practical usefulness of the three ML-Driven techniques, let us now compare the generated attacks patterns for the running example used in Section 6.1 It corresponds to one of the regular expressions that were actively protecting the industrial web service application used in our evaluation. Table 11 lists the attack patterns that were found in the same amount of time by ML-Driven E, ML-Driven B and ML-Driven D, respectively. As we can notice, ML-Driven E generated more attacks patterns compared to the other ML-Driven variants: ML-Driven $\mathrm{E}$ misses $p c_{5}$ and $p c_{8}$ while ML-Driven $\mathrm{D}$ further misses $p c_{2}$ and $p c_{9}$. Assuming the analyst would rely on ML-Driven B, she would add the following regular expression containing all found attack patterns: $r=$ $p c_{1}\left|p c_{2}\right| p c_{3}\left|p c_{4}\right| p c_{6}\left|p c_{7}\right| p c_{9}$, i.e., $r$ matches the attacks satisfying either $p c_{1}-p c_{4}, p c_{6}-p c_{7}$, or $p c_{9}$. However, this patch would miss attacks found by ML-Driven $\mathrm{E}$ as the generated $r$ would not match any attacks containing either $p c_{5}$ or $p c_{8}$. The patches generated with ML-Driven D would be less robust compared to ML-Driven E and ML-Driven B as the resulting regular expressions would not match attacks satisfying the missing four patterns.

The example above shows that generating more distinct attacks leads to uncovering more successful attack patterns and implementing more robust patches to the WAF.

\section{Discussion}

This section discusses some practical implications of our ML-Driven approach and its empirical results. .

\subsection{Differences between Case Studies}

When comparing the testing results of the two case studies some notable differences stand out. First, bypassing attacks could be found for each parameter protected by ModSecurity, whereas with the proprietary WAF, only 29 out of 75 parameters lead to bypassing attacks. This can be attributed to the fact that the latter strictly validates each input to follow an expected format. For example, a value provided to the parameter credit card number must consist of 16 to 19 digits and, otherwise, the request is rejected. SQL injection attacks typically require a larger character set and thus all attacks are blocked. Similarly, for the 46 parameters for which no attack is found, the expected input format prevents attacks. However, it is not possible to define such strict validation rules for all parameters, since the inputs might vary significantly in terms of character set and length. This is the case for the other 29 parameters where the expected input format is very general and the input validation rules rather loose, thus being prone to attacks. For example, the vulnerable parameter Address is expected to be a string with a maximum of 35 characters, a constraint with which many of the SQLi attacks comply.

Another major difference between the two case studies are the number of bypassing attacks per tested parameter. For ModSecurity, about 1,000 bypassing attacks per parameter are found while, for the proprietary WAF, they are on average 10,000. This significant difference can be attributed to the attack detection capabilities of each respective firewall and highlights the difficulty of customizing a rule set for a particular IT environment in practice. In our experiment, we use a default rule set for ModSecurity, while the proprietary WAF has a customized rule set to match a particular IT system. Such a customization is often necessary to achieve an acceptable false positive rate, but comes at the cost of reduced attack detection capabilities due in part to the lack of suitable tools to test the firewalls.

\subsection{Application of the Proposed Techniques}

We have proposed and evaluated three variants of a machine learning driven technique for the generation of SQLi attacks, namely ML-Driven E, ML-Driven $\mathrm{D}$, and ML-Driven B. ML-Driven D and ML-Driven B entail different strategies in allocating the test generation budget. ML-Driven E reconciles these differences and delivers a better performance. We have compared all these variants with RAN, the baseline technique considered in our work, on ModSecurity (a popular open-source WAF) and a proprietary WAF. Our experiments show that ML-Driven E outperforms all the other techniques.

We have also demonstrated the usefulness of mining more bypassing attacks in devising string patterns to fix WAFs. In our context, we experimented with RandomTree and RandomForest as machine classifiers for ML-Driven E. Since we show that the latter helps extract more path conditions that are useful in identifying patterns and fixing WAFs, we recommend the use of RandomForest.

\section{Conclusion}

Web application firewalls (WAFs) play an important role to protect online systems. The rising occurrence of new kinds of attacks and their increasing sophistication require that firewalls be updated and tested regularly, as otherwise attacks might remain undetected and reach the systems under protection.

We propose ML-Driven, a search-based approach that combines machine learning and evolutionary algorithms to automatically test the attack detection capabilities of WAFs. The approach automatically generates a diverse set of attacks, sends them to a WAF under test, and checks if they are correctly identified. By incrementally learning 
from the tests that are blocked or bypassing the firewall, our approach selects tests that exhibit string patterns with high bypassing probabilities (according the machine learning) and mutates them using an attack grammar designed to generate new and hopefully successful attacks. Identified bypassing attacks can be used to learn path conditions, which characterize successful attack patterns.

With such a set of bypassing attacks and path conditions that characterize them, a security expert can fix or fine-tune the WAF rules in order to block imminent SQLi attacks. In the attacker-defender war, time is vital. Being able to quickly learn and anticipate more attacks that can circumvent a firewall, in a timely manner, is very important to secure business data and services.

Though our approach was applied to SQL injection attacks in this paper, it can be adapted to other forms of attacks by making use of other attack grammars targeting different types of vulnerabilities.

Our key contributions in this work include (i) enhancing our preliminary techniques by consolidating them and improving their performance, (ii) comparing two different and adequate machine learning classifiers, (iii) carrying out a large-scale evaluation on two popular WAFs and (iv) comparing our approach with state-of-the-art tools. Evaluation results suggest that the performance of ML-Driven (and its enhanced variant in particular) is effective at generating many undetected attacks and provides a good basis to identify attack patterns to protect against. Further, it also fares significantly better than the best available tools.

In our future work, we will investigate automated approaches to generate effective patches for the WAF under test starting from the learned attack patterns. We reported on an initial attempt to automate the repairing process in a recent paper [11], where we generated patches that block as many bypassing attacks as possible while limiting the blocking of legitimate inputs. Investigating further, more effective, repairing strategies that better exploit the attack patterns generated by ML-Driven $\mathrm{E}$ is part of our future agenda. Finally, we plan to investigate various strategies (e.g., data re-sampling strategies, weighted training, and penalty- based training) to improve the effectiveness and the efficiency of ML-Driven by addressing the data imbalance problem.

\section{ACKNOWLEDGEMENTS}

This work builds on Dennis Appelt's Ph.D. dissertation. The project has received funding from the European Research Council (ERC) under the European Union's Horizon 2020 research and innovation programme (grant agreement No 694277).

\section{References}

[1] Verified firewall policy transformations for test case generation. In Software Testing, Verification and Validation (ICST), 2010 Third International Conference on, pages 345-354. IEEE, 2010.

[2] Management of an Academic HPC Cluster: The UL Experience. In Proc. of the 2014 Intl. Conf. on High Performance Computing $\mathcal{E}$ Simulation (HPCS 2014), pages 959-967, Bologna, Italy, July 2014. IEEE.

[3] E. Al-Shaer, A. El-Atawy, and T. Samak. Automated pseudo-live testing of firewall configuration enforcement. Selected Areas in Communications, IEEE Journal on, 27(3):302-314, 2009.
[4] S. Anand, E. K. Burke, T. Y. Chen, J. Clark, M. B. Cohen, W. Grieskamp, M. Harman, M. J. Harrold, and P. McMinn. An orchestrated survey of methodologies for automated software test case generation. Journal of Systems and Software, 86(8):1978-2001, 2013.

[5] N. Antunes, N. Laranjeiro, M. Vieira, and H. Madeira. Command injection vulnerability scanner for web services. http://eden.dei.uc.pt/ mvieira/.

[6] N. Antunes, N. Laranjeiro, M. Vieira, and H. Madeira. Effective detection of SQL/XPath injection vulnerabilities in web services. In Proceedings of the 6th IEEE International Conference on Services Computing (SCC '09), pages 260-267, 2009.

[7] D. Appelt, N. Alshahwan, and L. Briand. Assessing the impact of firewalls and database proxies on sql injection testing. In Proceedings of the 1st International Workshop on Future Internet Testing, 2013.

[8] D. Appelt, A. Nguyen, Cu D. Panichella, and L. Briand. Automated testing of web application firewalls: Technical report. Technical Report TR-SnT-2016-1, University of Luxembourg, 2016.

[9] D. Appelt, C. D. Nguyen, and L. Briand. Behind an application firewall, are we safe from sql injection attacks? In Software Testing, Verification and Validation (ICST), 2015 IEEE 8th International Conference on, pages 1-10. IEEE, 2015.

[10] D. Appelt, C. D. Nguyen, L. C. Briand, and N. Alshahwan. Automated testing for sql injection vulnerabilities: An input mutation approach. In Proceedings of the 2014 International Symposium on Software Testing and Analysis, ISSTA 2014, pages 259-269, New York, NY, USA, 2014. ACM.

[11] D. Appelt, A. Panichella, and L. C. Briand. Automatically repairing web application firewalls based on successful SQL injection attacks. In 28th IEEE International Symposium on Software Reliability Engineering, ISSRE 2017, Toulouse, France, October 23-26, 2017, pages 339-350, 2017.

[12] W. Banzhaf, F. D. Francone, R. E. Keller, and P. Nordin. Genetic Programming: An Introduction: on the Automatic Evolution of Computer Programs and Its Applications. Morgan Kaufmann Publishers Inc., San Francisco, CA, USA, 1998.

[13] J. Bau, E. Bursztein, D. Gupta, and J. Mitchell. State of the art: Automated black-box web application vulnerability testing. In Security and Privacy (SP), 2010 IEEE Symposium on, pages 332-345. IEEE, 2010.

[14] S. W. Boyd and A. D. Keromytis. Sqlrand: Preventing sql injection attacks. In Applied Cryptography and Network Security, pages 292302. Springer, 2004.

[15] L. Breiman. Random forests. Machine learning, 45(1):5-32, 2001.

[16] J. Coffey, L. White, N. Wilde, and S. Simmons. Locating software features in a soa composite application. In Web Services (ECOWS), 2010 IEEE 8th European Conference on, pages 99-106, 2010.

[17] L. Desmet, F. Piessens, W. Joosen, and P. Verbaeten. Bridging the gap between web application firewalls and web applications. In Proceedings of the fourth ACM workshop on Formal methods in security, pages 67-77. ACM, 2006.

[18] A. Doupé, M. Cova, and G. Vigna. Why johnny canâĂŹt pentest: An analysis of black-box web vulnerability scanners. In International Conference on Detection of Intrusions and Malware, and Vulnerability Assessment (DIMVA), pages 111-131. Springer, 2010.

[19] M. Felderer, M. BÃijchler, M. Johns, A. D. Brucker, R. Breu, and A. Pretschner. Security testing. Advances in Computers, 101:1 - 51, 2016.

[20] X. Fu, X. Lu, B. Peltsverger, S. Chen, K. Qian, and L. Tao. A static analysis framework for detecting sql injection vulnerabilities. In 31st Annual International Computer Software and Applications Conference (COMPSAC 2007), volume 1, pages 87-96, July 2007.

[21] P. Godefroid, A. Kiezun, and M. Y. Levin. Grammar-based whitebox fuzzing. In ACM Sigplan Notices, volume 43, pages 206-215. ACM, 2008.

[22] P. Godefroid, M. Y. Levin, and D. Molnar. Sage: whitebox fuzzing for security testing. Queue, 10(1):20, 2012.

[23] P. Godefroid, M. Y. Levin, D. A. Molnar, et al. Automated whitebox fuzz testing. In NDSS, volume 8, pages 151-166, 2008.

[24] W. Halfond, J. Viegas, and A. Orso. A classification of sqlinjection attacks and countermeasures. In Proceedings of the IEEE International Symposium on Secure Software Engineering, volume 1, pages 13-15. IEEE, 2006.

[25] W. G. Halfond, S. Anand, and A. Orso. Precise interface identification to improve testing and analysis of web applications. In Proceedings of the 18th International Symposium on Software Testing and Analysis (ISSTA '09), pages 285-296, 2009. 
[26] W. G. Halfond and A. Orso. Amnesia: analysis and monitoring for neutralizing sql-injection attacks. In Proceedings of the 20th IEEE/ACM international Conference on Automated software engineering, pages 174-183. ACM, 2005.

[27] W. G. J. Halfond and A. Orso. Preventing SQL injection attacks using AMNESIA. In Proceedings of the 28th International Conference on Software Engineering (ICSE' 06), pages 795-798, 2006.

[28] M. Hall, E. Frank, G. Holmes, B. Pfahringer, P. Reutemann, and I. H. Witten. The weka data mining software: An update. SIGKDD Explor. Newsl., 11(1):10-18, Nov. 2009.

[29] J. Hwang, T. Xie, F. Chen, and A. X. Liu. Systematic structural testing of firewall policies. In Reliable Distributed Systems, 2008. SRDS'08. IEEE Symposium on, pages 105-114. IEEE, 2008.

[30] J. Jürjens and G. Wimmel. Specification-based testing of firewalls. In D. Bjørner, M. Broy, and A. Zamulin, editors, Perspectives of System Informatics, volume 2244 of Lecture Notes in Computer Science, pages 308-316. Springer Berlin Heidelberg, 2001.

[31] G. Karafotias, M. Hoogendoorn, and A. E. Eiben. Parameter control in evolutionary algorithms: Trends and challenges. IEEE Transactions on Evolutionary Computation, 19(2):167-187, April 2015.

[32] A. Kieyzun, P. J. Guo, K. Jayaraman, and M. D. Ernst. Automatic creation of SQL injection and cross-site scripting attacks. In Proceedings of the 31st International Conference on Software Engineering (ICSE '09), pages 199-209, 2009.

[33] Y.-F. Li, P. K. Das, and D. L. Dowe. Two decades of web application testingâĂŤa survey of recent advances. Information Systems, 43:20 $-54,2014$.

[34] A. Liu, Y. Yuan, D. Wijesekera, and A. Stavrou. Sqlprob: A proxybased architecture towards preventing sql injection attacks. In Proceedings of the 2009 ACM Symposium on Applied Computing, SAC '09, pages 2054-2061, New York, NY, USA, 2009. ACM.

[35] H. Liu and H. B. Kuan Tan. Testing input validation in web applications through automated model recovery. Journal of Systems and Software, 81(2):222-233, 2008.

[36] P. McMinn. Search-based software test data generation: a survey. Software Testing, Verification and Reliability, 14(2):105-156, 2004.

[37] R. McNally, K. Yiu, D. Grove, and D. Gerhardy. Fuzzing: the state of the art. Technical report, DTIC Document, 2012.

[38] J. Offutt, Y. Wu, X. Du, and H. Huang. Bypass testing of web applications. In Software Reliability Engineering, 2004. ISSRE 2004. 15th International Symposium on, pages 187-197. IEEE, 2004.
[39] A. Panichella, F. Kifetew, and P. Tonella. Automated test case generation as a many-objective optimisation problem with dynamic selection of the targets. IEEE Transactions on Software Engineering, PP(99):1-1, 2017.

[40] A. Panichella, F. M. Kifetew, and P. Tonella. Reformulating branch coverage as a many-objective optimization problem. In 8th IEEE International Conference on Software Testing, Verification and Validation, ICST 2015, Graz, Austria, April 13-17, 2015, pages 1-10, 2015.

[41] A. Petrowski and S. Ben-Hamida. Evolutionary Algorithms. John Wiley \& Sons, 2017.

[42] J. R. Quinlan. C4.5: Programs for Machine Learning, volume 1. Morgan kaufmann, 1993.

[43] D. Senn, D. Basin, and G. Caronni. Firewall conformance testing. In F. Khendek and R. Dssouli, editors, Testing of Communicating Systems, volume 3502 of Lecture Notes in Computer Science, pages 226-241. Springer Berlin Heidelberg, 2005.

[44] S. Shamshiri, J. M. Rojas, G. Fraser, and P. McMinn. Random or genetic algorithm search for object-oriented test suite generation? In Proceedings of the 2015 Annual Conference on Genetic and Evolutionary Computation, GECCO '15, pages 1367-1374, New York, NY USA, 2015. ACM.

[45] L. K. Shar and H. B. K. Tan. Defeating sql injection. Computer, (3):69-77, 2013.

[46] M. Soltani, A. Panichella, and A. van Deursen. A guided genetic algorithm for automated crash reproduction. In Proceedings of the 39th International Conference on Software Engineering, ICSE 2017, Buenos Aires, Argentina, May 20-28, 2017, pages 209-220, 2017.

[47] M. Sutton, A. Greene, and P. Amini. Fuzzing: brute force vulnerability discovery. Pearson Education, 2007.

[48] O. Tripp, O. Weisman, and L. Guy. Finding your way in the testing jungle: a learning approach to web security testing. In Proceedings of the 2013 International Symposium on Software Testing and Analysis, pages 347-357. ACM, 2013.

[49] M. Crepinšek, S.-H. Liu, and M. Mernik. Exploration and exploitation in evolutionary algorithms: A survey. ACM Comput. Surv., $45(3), 2013$

[50] J. Williams and D. Wichers. Owasp, top 10, the ten most critical web application security risks. Technical report, The Open Web Application Security Project, 2013.

[51] I. H. Witten and E. Frank. Data Mining: Practical machine learning tools and techniques. Morgan Kaufmann, 2011. 